\title{
The riparian flora and plant communities of the Pilbara region of Western Australia
}

\author{
M.N. Lyons \\ Department of Parks and Wildlife, Science and Conservation Division, \\ Kieran McNamara Conservation Science Centre, Locked Bag 104, Bentley Delivery Centre, \\ Western Australia 6983, Australia. Email: mike.lyons@dpaw.wa.gov.au
}

\begin{abstract}
A survey of riparian flora and plant communities was undertaken at 98 wetlands and rivers in the Pilbara region of Western Australia. Sampling was quadrat-based, with floristics, surface soils and wetland attributes recorded. Selected sites captured the full range of Pilbara wetland types including springs, river pools, claypans, salt marshes and rock pools. A total of 455 taxa was recorded from the survey sites, representing ca. $25 \%$ of the known flora of the Pilbara bioregion. The flora is dominated by taxa with Eremaean and tropical affinities, with only six taxa endemic in the region. Of recorded taxa known from four or fewer bioregions, most are shared with the adjacent Carnarvon and Gascoyne bioregions rather than the adjoining internally draining deserts. Sixteen taxa of conservation significance were documented, with claypans, the Fortescue Marsh, and Millstream and Karijini National Park sites dominating occurrences of rare species. Eight major groups were defined by classifying wetlands in terms of species presence/absence data. Floristic patterning was strongly aligned with the major wetland types (geomorphic/hydrological) used in the primary sampling stratification. A combination of wetland morphology/hydrological setting, site edaphic attributes and distance to the coast were dominant variables related to riparian floristic composition. Primary compositional separation was observed between riverine and non-riverine sites, with lowland turbid riverine sites with finetextured soils compositionally related to claypans and clay flats. Limited biogeographic patterning was evident except where individual IBRA subregions and drainage basins were dominated by few wetland types.
\end{abstract}

Keywords - Pilbara, wetland, riparian, floristic composition, botanical survey, vegetation, rivers

\section{INTRODUCTION}

The Pilbara IBRA region of Western Australia is a region of approximately $179,000 \mathrm{~km}^{2}$ that corresponds broadly with the Pilbara Craton, a major geological block of Archaean origin (Geological Survey of Western Australia 1990). The region is recognised as biogeographically distinct under the scheme developed by Thackway and Creswell (1994), based on the original Fortescue Botanical District defined by Beard (1990). A detailed description of the soils, climate and landforms of the region is provided by McKenzie et al. (2011).

The region's climate is broadly arid, with highly seasonal, typically intense, summer rainfall that may include cyclonic systems (Leighton 2004). This, coupled with a rocky middle and upper landscape, produces river flows of high energy and volume and relatively short duration. Extensive aquifer systems discharge at the surface to form springs and contribute perennial flows to rivers and creeks. In lowlands, major rivers have broad channels with flood flows forming numerous side channels. Clay flats, claypans and lowland creeks with fine sediments are typically highly turbid.

Land use in the region is overwhelmingly dominated by mining and extensive pastoral use. These uses place pressures on Pilbara wetlands through hydrological changes associated with mine dewatering and discharges, and the impact of grazing cattle. In the Pilbara, stock watering at natural water points is uncontrolled, concentrating trampling and grazing in riparian zones, leading to 


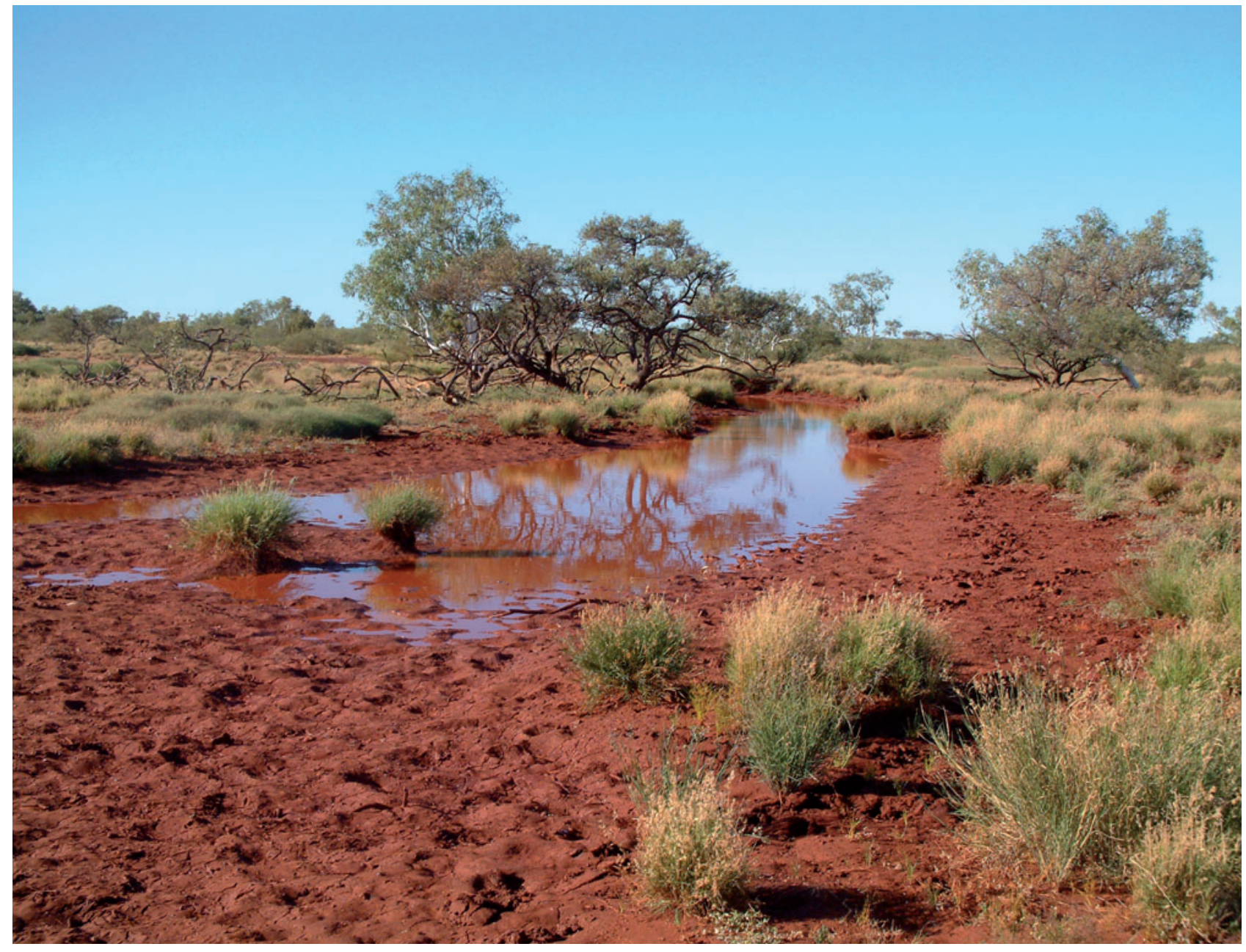

Plate 1 A highly turbid ephemeral claypan at Yarraloola (site PSW074A) in lowlands dominated by Eriachne benthamii. Trampling by cattle is evident (M.N. Lyons).

bank erosion and waterbody siltation. Aggregation of stock, particularly late in the dry season, leads to the eutrophication of waterbodies from accumulated faeces.

Protection of wetland biological values across the Pilbara is contingent on a greater understanding of their biogeographic patterning, for a number of their biotic components. The existing reserve network in the Pilbara consists of four reserves that, while capturing some major wetlands (e.g. Karijini National Park - gorges, Millstream National Park - springs), do not include the full diversity of Pilbara wetlands. The inclusion of wetland floristic data to inform formal conservation planning is a relatively recent feature of broadscale biodiversity survey in Western Australia (Keighery et al. 2000; Lyons et al. 2004; Walshe et al. 2004). This paper aims to document the riparian flora of the wetlands and rivers of the Pilbara and examine how the major geographic and site environments correlate with floristic composition. The data sets and understanding developed in the current study, coupled with studies of aquatic invertebrates by Pinder et al. (2011), will provide the basis for spatially explicit modelling of the biotic composition of Pilbara wetlands and rivers.

\section{STUDY AREA AND METHODS}

The Pilbara region as defined in this study is bounded to the south by the Ashburton River and the east by the De Grey-Oakover River system. A small number of wetlands were also sampled in the Rudall River area to the east of the main study area (Figure 1). The study area includes the entire Pilbara IBRA region (Thackway and Cresswell 1994) and additional small areas of the adjoining Gascoyne and Little Sandy Desert regions, representing a total area of approximately $225,000 \mathrm{~km}^{2}$.

Precipitation across the region is dominated by summer rainfall associated with tropical lowpressure systems producing thunderstorms and cyclones. Annual average rainfall is $290 \mathrm{~mm}$. 

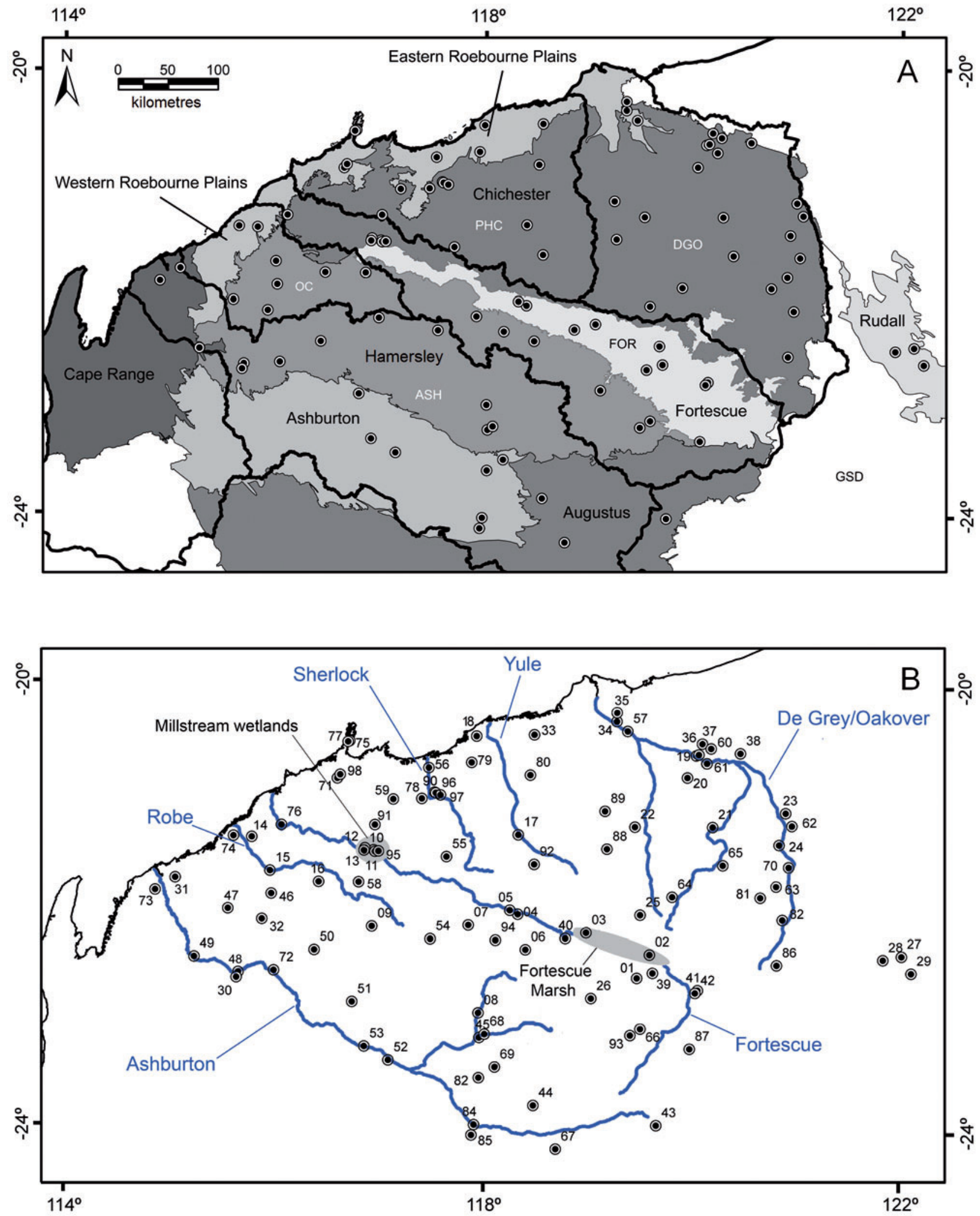

Figure 1 Maps of the Pilbara study area showing the 98 wetland sites sampled (note A and B sites at the same wetland are shown as a single point). Figure 1A shows IBRA6.1 subregions as shaded areas with full names; the Pilbara Bioregion comprises four subregions: Roebourne Plains (Eastern \& Western), Chichester, Fortescue and Hamersley. Drainage basin boundaries are outlined in black and labelled with abbreviated names as follows: ASH, Ashburton; OC, Onslow Coast; FOR, Fortescue; PHC, Port Hedland Coast; DGO, De Grey-Oakover; GSD, Great Sandy Desert. Figure 1B shows the main rivers in blue and two major wetlands (shaded grey). The sampled wetland sites are numbered as per Table 1. 
Inter-annually and spatially, rainfall variability is high, driven largely by the occurrence and path of cyclones. Winter rainfall from cold fronts originating in the south contributes significantly in the western coastal and central uplands of the region, and attenuates markedly towards the east. Two resulting bioclimatic zones were identified by Beard (1990), with the western coastal area characterised as semi-desert tropical, and the remainder as desert.

\section{Site selection and sampling}

Ninety-eight wetlands were selected to capture the diversity of wetland types present in each of the five major drainage basins within the Pilbara (Figure 1A, Table 1). The selected wetlands formed the basis of an integrated wetland survey of the Pilbara that covered a variety of biotic groups of the water column, including the riparian flora and vegetation reported here (see McKenzie et al. 2009, Pinder et al. 2010). Here, riparian is used to describe environments at the edge of waterbodies; i.e. under the hydrological influence of the wetland but not inundated at the time of sampling. This includes river banks, the waterlogged shallow soil aprons of rock pools, and the margins of claypans. Some riverine communities, such as Melaleuca glomerata on sandy islands, were rarely sampled since they were often not adjacent to waterbodies.

The primary stratification employed in the survey was the a priori designation of a number of wetland types based on hydrological and morphological attributes. These closely follow the scheme adopted for the region by Masini (1998): rock pools; springs and their outflow creeks; river pools; turbid linear pools; claypans and flooded clay flats; ephemeral creeks; salt marshes; and gorges. Wetlands were selected by examining topographic maps and consulting local experts, followed by aerial and ground-based reconnaissance. Within major river systems, sampling sites were distributed along the length of the main channel, and included the full range of stream orders from ephemeral headwater creeks to near-coastal river pools (Figure 1B).

Sampling was undertaken within a representative section within each selected wetland. At most wetlands (93), a single linear quadrat $\left(200 \mathrm{~m}^{2}\right)$ was established parallel to the waterbody margin and positioned to capture the typical riparian setting of the wetland. Typically, quadrat configurations at each wetland were a single $4 \mathrm{~m} \times 50 \mathrm{~m}$ or $5 \mathrm{~m} \mathrm{x}$ $40 \mathrm{~m}$ rectangle, depending on riparian zone width. Within each quadrat, all vascular plants were scored within eight contiguous $25 \mathrm{~m}^{2}$ subquadrats to evaluate the adequacy of quadrat size. Two $200 \mathrm{~m}^{2}$ quadrats (designated A and B, see Table

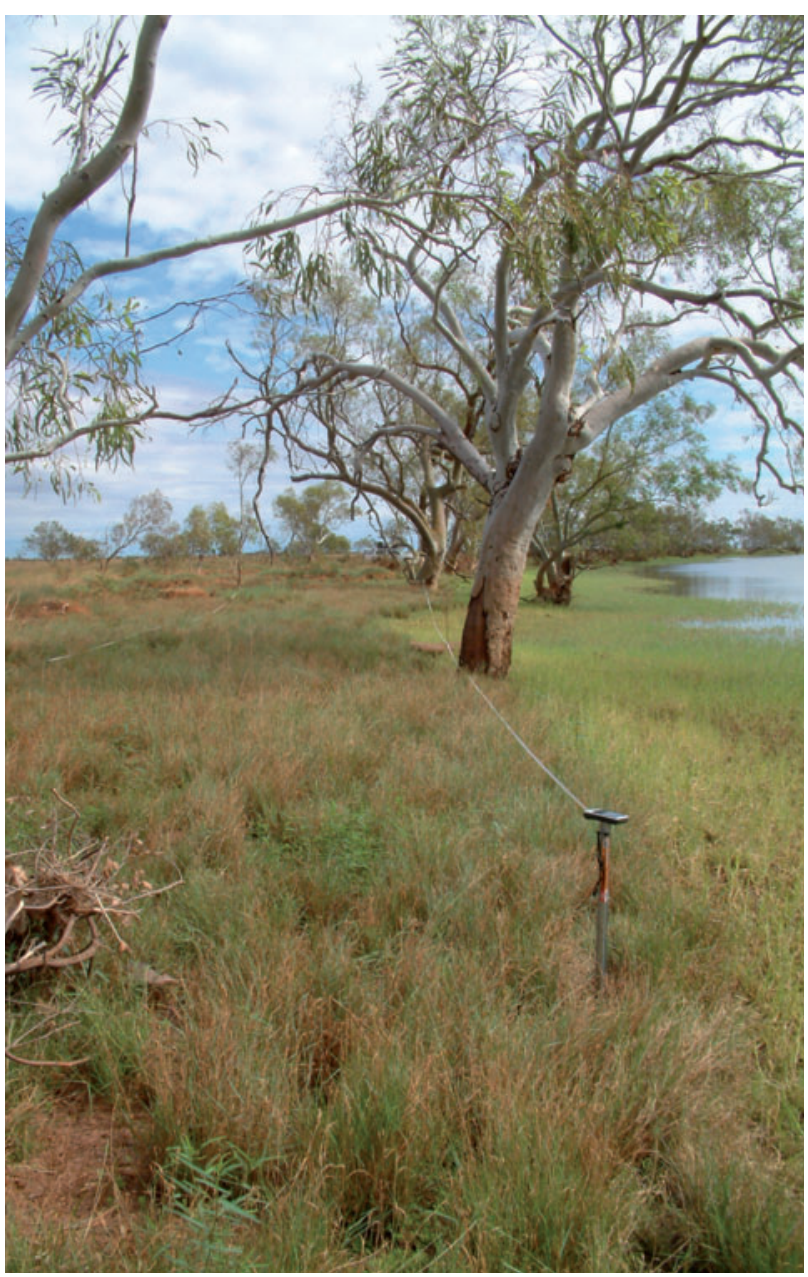

Plate 2 Munreemya Billabong, a seasonal/episodic wetland on Yarrie Station (wetland site PSW036A). The riparian zone dominated by Eucalyptus camaldulensis and E. victrix over Eriachne benthamii and Dichanthium fecundum sampled using a linear quadrat (M.N. Lyons).

1) were sampled at each of five large wetlands (PSW002, PSW003, PSW004, PSW035, and PSW080), to capture the major wetland zonation. At some small wetlands (typically small claypans and ephemeral streams), discontiguous subquadrats were surveyed in order to cumulatively sample 200 $\mathrm{m}^{2}$ and to avoid upland vegetation. Quadrats were marked with steel pegs and their locations recorded using handheld GPS. For wetlands where the entire riparian zone was less than $200 \mathrm{~m}^{2}$, such as very small claypans and rock pools with fringing shallow soil aprons, the whole wetland fringe was sampled (Table 1). These different quadrat configurations are herein referred to as sites. Sites were scored twice, in autumn and spring, between 2004 and 2006. 


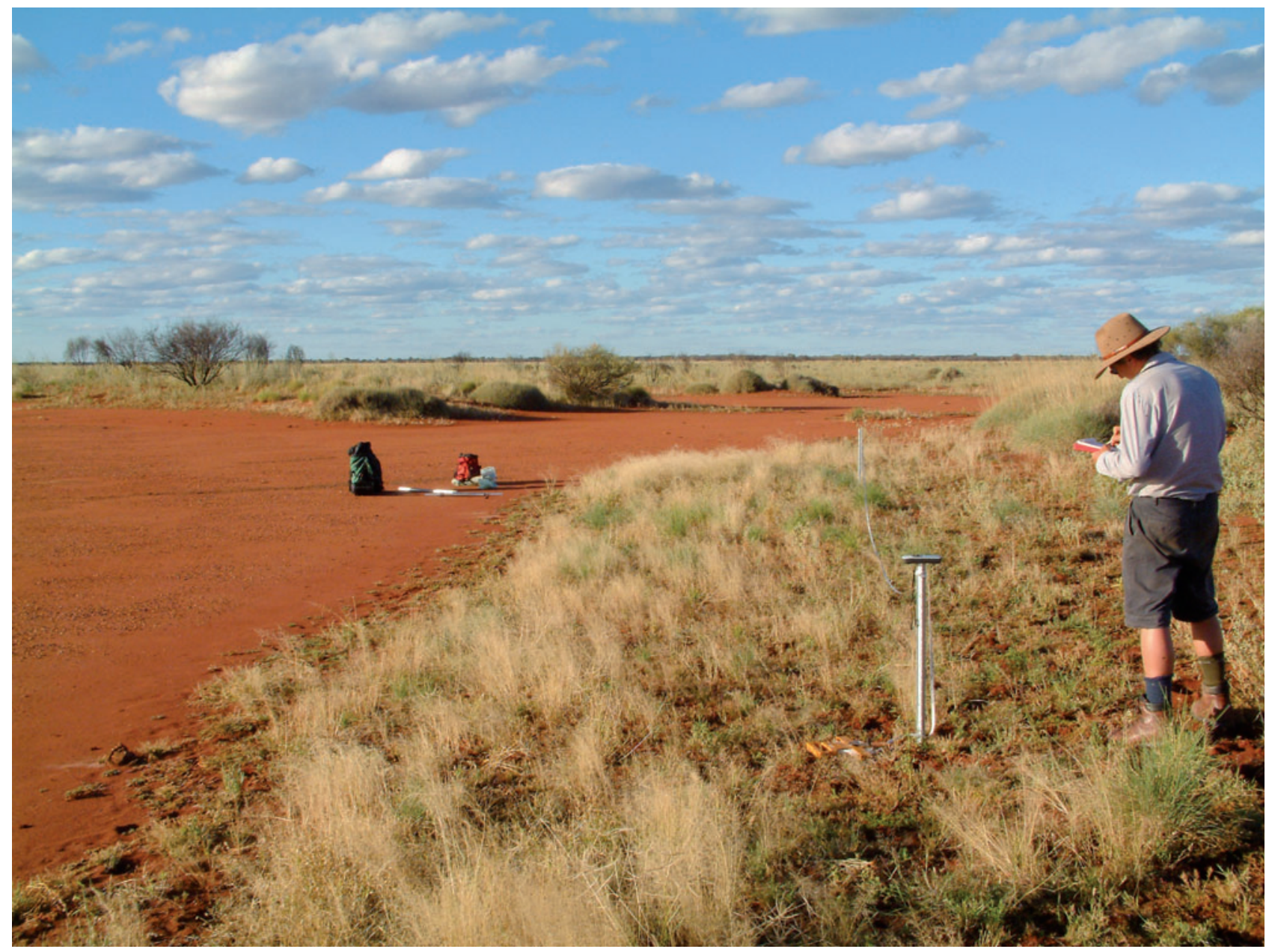

Plate 3 Ephemeral claypan on Roy Hill Station (site PSW039A). Sampling the grass- and herb-rich margin downslope of upland vegetation dominated by Triodia (D. Mickle).

Species accumulation curves were produced in EstimateS (using Mao Tau) for eight sites to examine the adequacy of the quadrat design (Colwell 2009). Input data were compiled as a presence/absence matrix of species at the eight subquadrats $\left(25 \mathrm{~m}^{2}\right)$ within each $200 \mathrm{~m}^{2}$ quadrat.

Within sites, surface soil samples (at 5-15 cm depth) were collected from 30 spaced points and bulked in the field to yield approximately $2.5 \mathrm{~kg}$ samples. Particle size and soil chemistry were analysed by the Chemistry Centre of Western Australia. Parameters analysed included $\mathrm{pH}$, electrical conductivity, organic carbon, phosphorous, nitrogen, potassium, calcium, magnesium, sodium, and percent silt, clay and sand, and three gravel fractions (Table 2). A number of additional variables were derived for the sites by Pinder et al. (2011) and are used here. They include Strahler stream order (Strahler 1952), four inferred permanence/hydroperiod classes $(1=$ ephemeral, 2 = seasonal episodic, $3=$ near permanent, $4=$ permanent), straight line distance to coast, riverine versus non-riverine sites, and altitude (Table 3). A suite of climatic variables (Table 3) was derived from the BIOCLIM module of ANUCLIM (Houlder et al. 2000).

To provide a broader perspective on the riparian flora, the proportion it represents of the known Pilbara flora, based on records held at the Western Australian Herbarium (S. Dillon, unpublished data), was determined. Additionally, the broader biogeographic relationships of the riparian flora were explored by examining the IBRA regional occurrences of the taxa across Western Australia based on voucher specimen geocodes. Due to differences in taxonomic resolution across the data sets, some taxa were amalgamated at the specific level to yield a final dataset of 430 taxa across the State's IBRA regions. Data on the occurrence of Pilbara riparian taxa were also compiled for the botanical provinces (Northern, Eremaean and South-West) of Beard (1990), treating the Coolgardie IBRA region as Eremaean. 


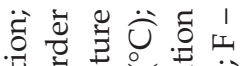
चु ₹

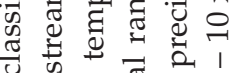
뎨

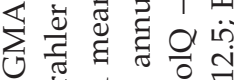
के

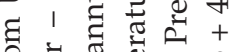
我 1 号 उ के द्वि ह ह \& $\sigma$ क 象 席造

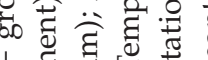
1 医

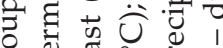

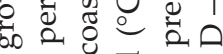

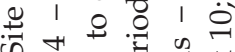
क स

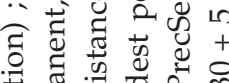
跣语

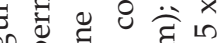

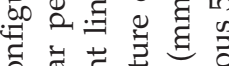

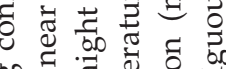

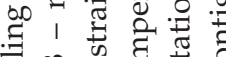
का का है 氜 क्ष

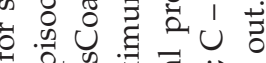

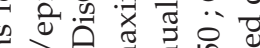

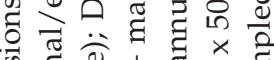
क्ष कृ क्षे की 1 की

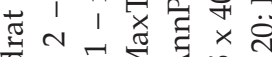
证云的 るั $\checkmark$ च 0 -

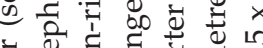
ए 1 व $=1$ ส च क 웡 क ज 3 过

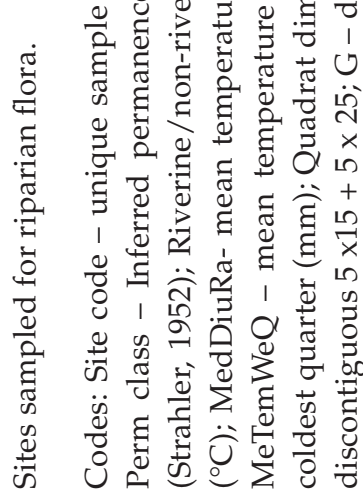

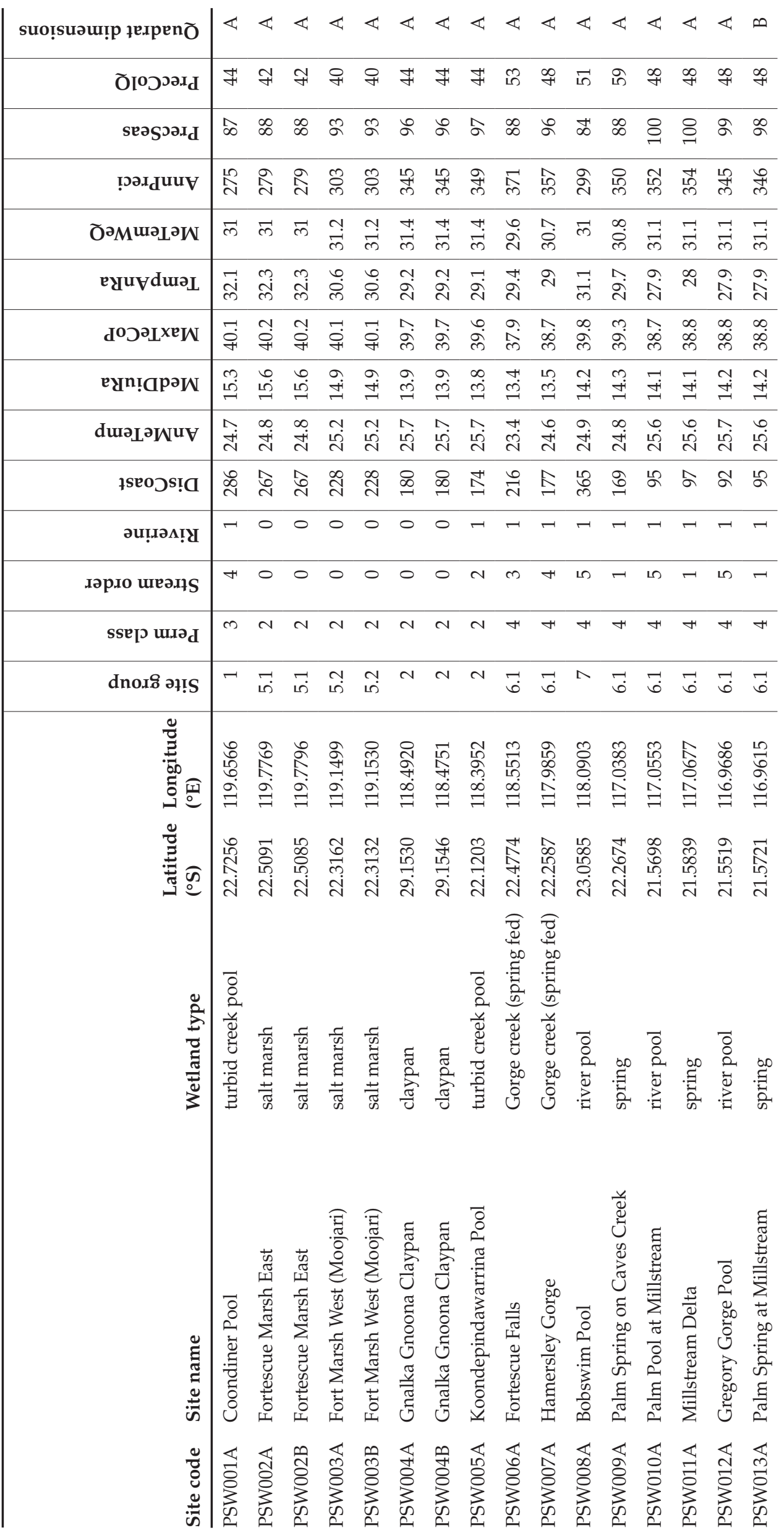




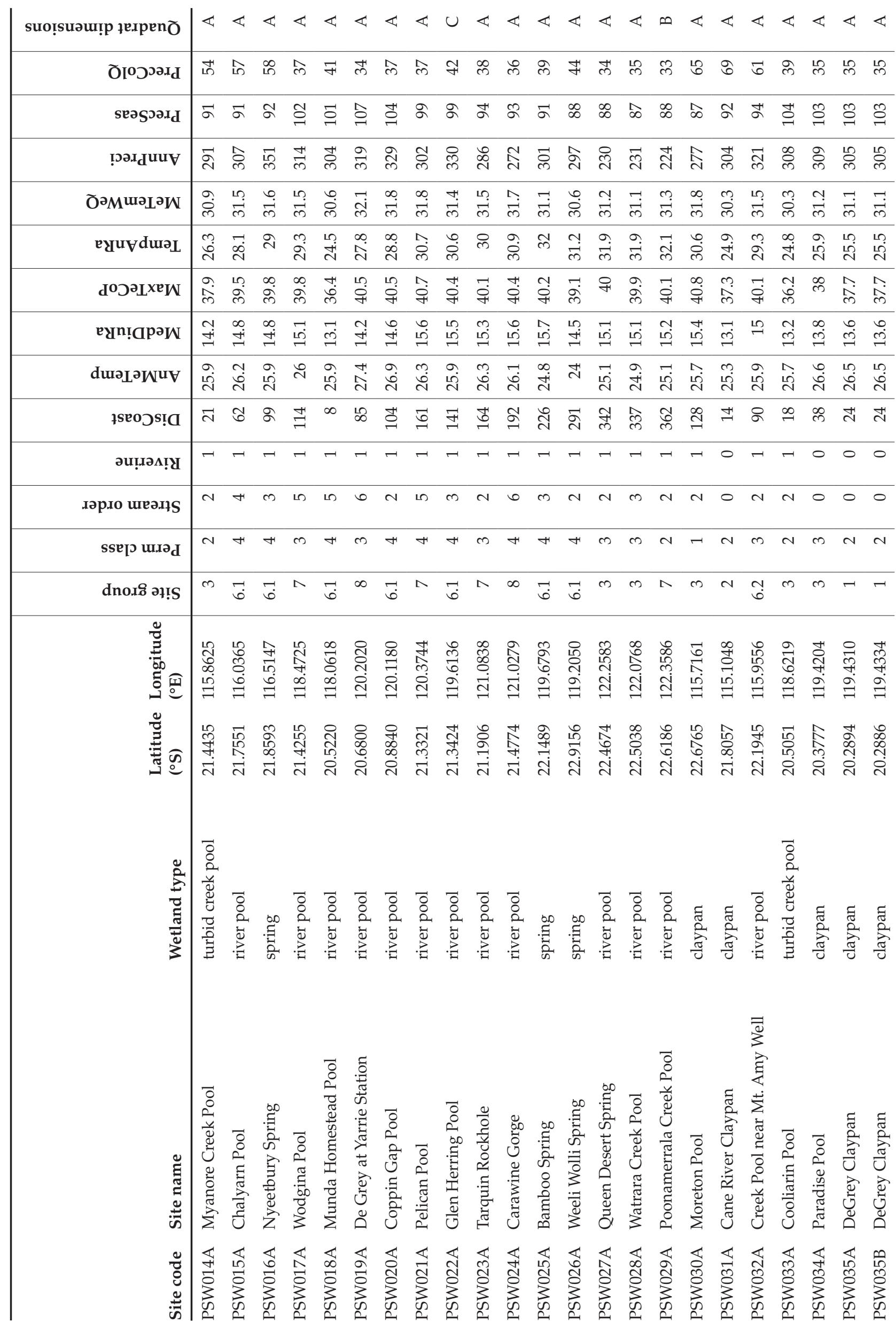




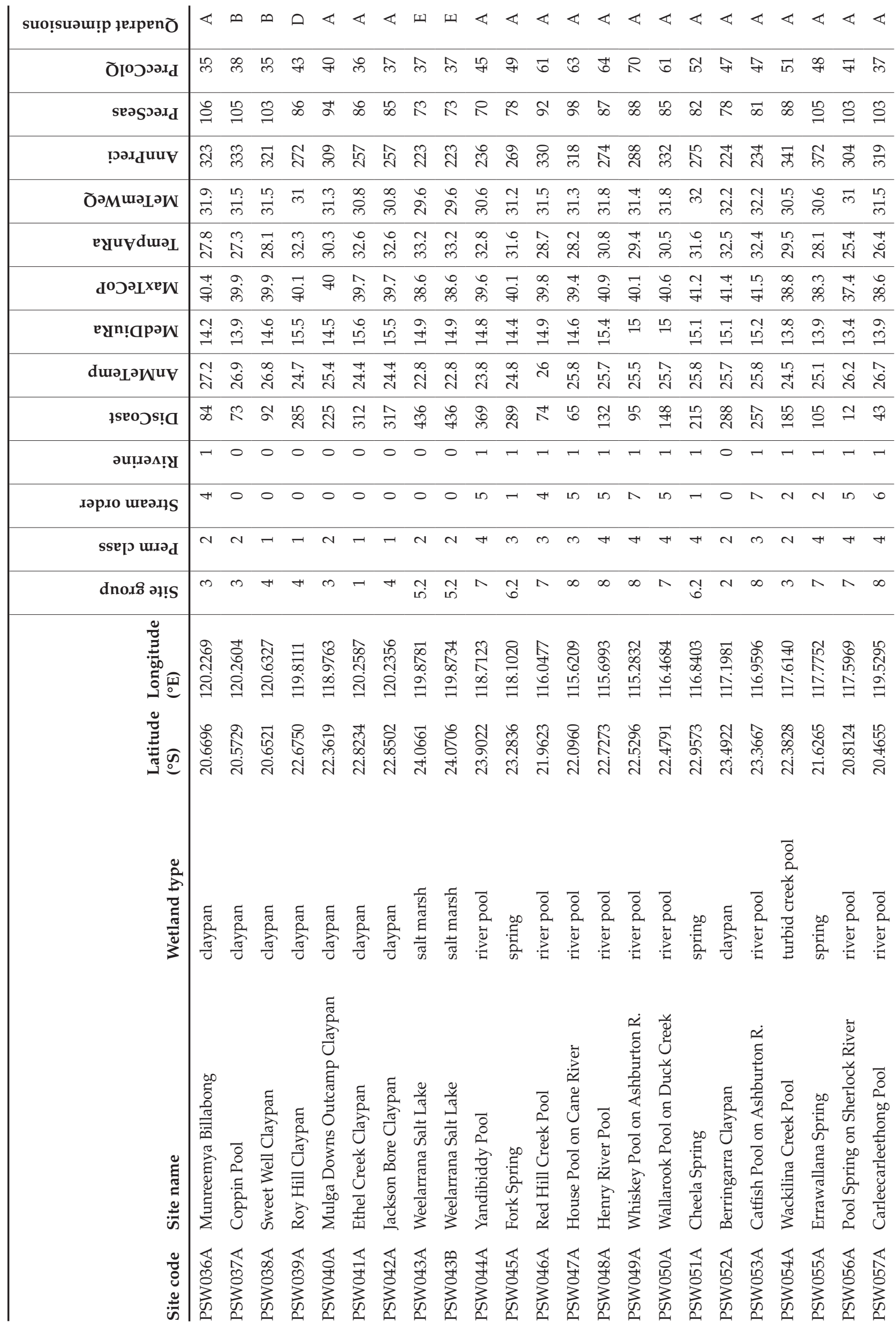




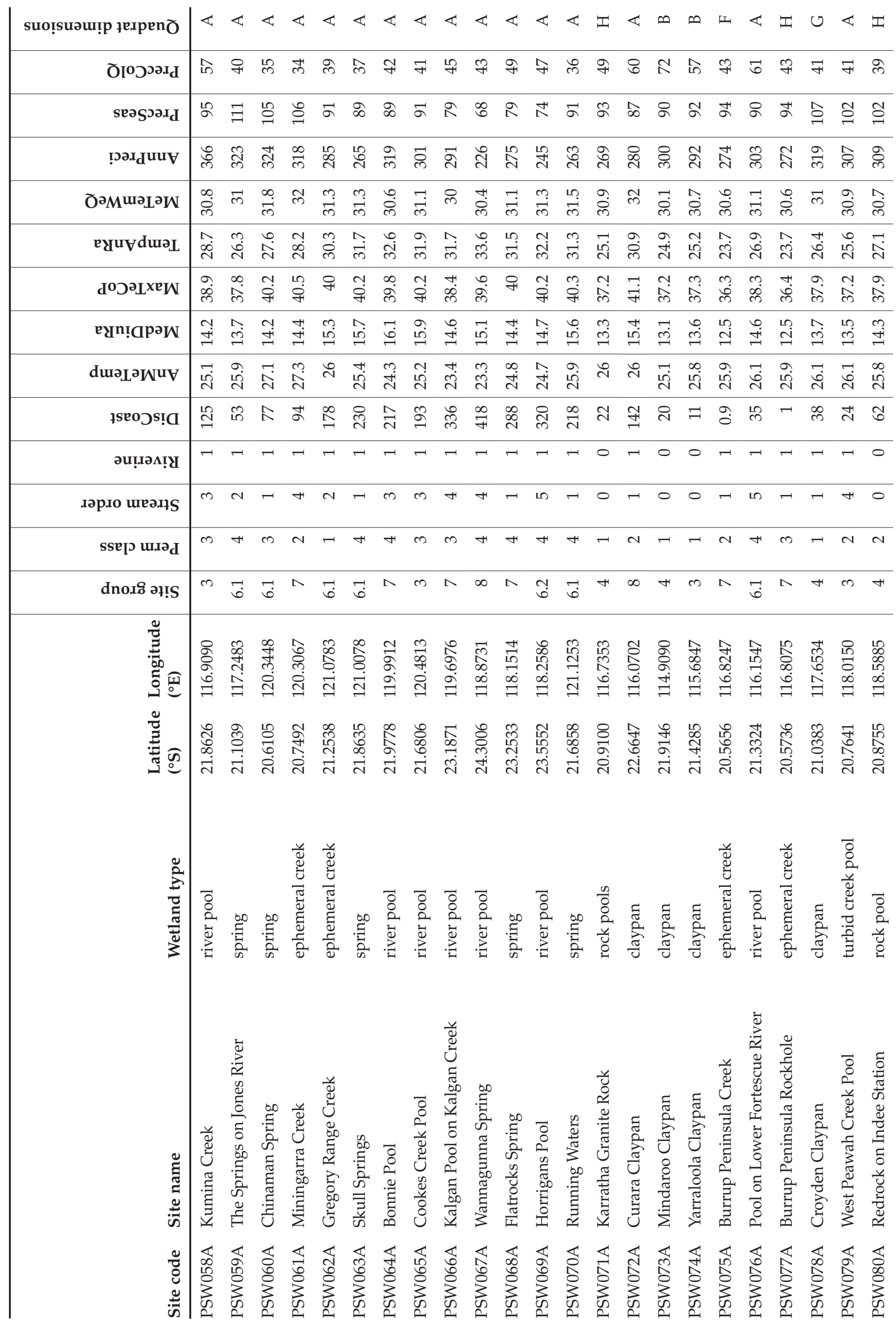




\begin{tabular}{|c|c|c|c|c|c|c|c|c|c|c|c|c|c|c|c|c|c|c|c|}
\hline suo!̣suau!̣p łexpenð & $\Psi \ll$ & $\varangle$ & ค & $\varangle$ & & & & & & & & & $\mathbb{E}$ & & & & & & $\ll \ll$ \\
\hline 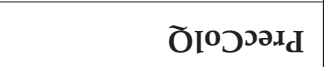 & ले ले & m & f & டி & กิ & लै & & & & & क्ष & f & ले & Fi & & & t & F & F Fे \\
\hline seasoax & 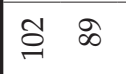 & $\infty$ & $\mathbb{N}$ & $\infty$ & 6 & $\infty$ & & & & & $\overbrace{}^{\infty}$ & $\stackrel{2}{2}$ & \& & 2 & & & & & $\stackrel{\circ}{\circ}$ \\
\hline 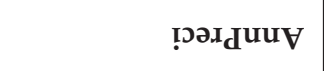 & 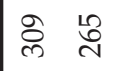 & 今 & శ్సి & 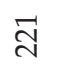 & సิ & ल्సి & & & & & ले & 芯 & $\stackrel{m}{m}$ & సે & & & $p$ & ले & $\stackrel{\circ}{\vec{c}}$ \\
\hline бәМшәцәИ & 仓ิ & $\vec{m}$ & 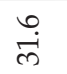 & $\stackrel{\llcorner}{m}$ & हें & हें & & & & & s. & : & $\stackrel{n}{\vec{m}}$ & Әे & & & 5 & $\vec{m}$ & \begin{tabular}{ll}
\cline { 1 - 1 } & $\infty$ \\
$\dot{m}$ & $\stackrel{m}{m}$
\end{tabular} \\
\hline eyuydurə & तิ लै & $\overrightarrow{\text { ते }}$ & $\begin{array}{l}\text { L } \\
\text { ले }\end{array}$ & $\overrightarrow{\ddot{\infty}}$ & $\ddot{m}$ & तु & $\infty$ & $g^{2}$ & & & t: & సે & के & $\stackrel{\text { m. }}{m}$ & & & 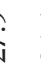 & ث̈. & 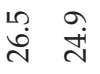 \\
\hline $\mathbf{d}^{0} J^{\partial} L^{x e} \mathbf{W}$ & 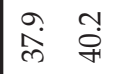 & f & 今̀ & $\hat{o}$ & 年 & ळें & $\begin{array}{l}\infty \\
\infty \\
\infty \\
\infty\end{array}$ & q & & $?$ & के & 离 & 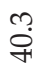 & $\begin{array}{l}-1 \\
\infty \\
\infty\end{array}$ & d & & 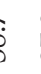 & $\frac{5}{n}$ & 항 \\
\hline еуп!арәш & $\begin{array}{l}\stackrel{\infty}{\sharp} \\
\stackrel{1}{+}\end{array}$ & $\begin{array}{l}\text { மீ } \\
\stackrel{\text { Lे }}{n}\end{array}$ & $\stackrel{\infty}{\sim}$ & $\stackrel{\llcorner 2}{\sim}$ & $\stackrel{\vartheta}{ \pm}$ & 总 & $\stackrel{20}{\sim}$ & 7 & & & \begin{tabular}{l}
0 \\
\multicolumn{2}{c}{} \\
$\vec{c}$
\end{tabular} & $\begin{array}{l}\infty \\
\stackrel{\sim}{\rightarrow}\end{array}$ & 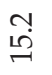 & $\stackrel{\llcorner}{+}$ & ch & & & $\vec{c}$ & 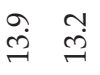 \\
\hline duә & 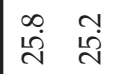 & 롬 & 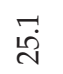 & $\stackrel{\circlearrowright}{\stackrel{0}{N}}$ & $\stackrel{+}{i}$ & $\stackrel{0}{\stackrel{0}{N}}$ & त & $\stackrel{2}{c}$ & & 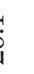 & فे & ํํㄹ & में & $\overrightarrow{\ddot{\sim}}$ & C & & to & فे & స్ֶ \\
\hline łseoวs!a & તెర & $\underset{N}{\mathbb{N}}$ & ले & $\stackrel{\infty}{\infty}$ & $\underset{\infty}{\infty}$ & 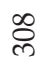 & ભ & 5 & 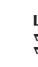 & 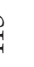 & F & $R$ & 告 & $\stackrel{m}{m}$ & ¿ุ & & R & e & F \\
\hline әчџџәц!Х & $0 \quad r$ & 0 & $r$ & $\neg$ & - & - & - & & & & T & - & - & - & & & 4 & -1 & $\rightarrow 0$ \\
\hline ләр.о шеәня & $N$ & in & 0 & 0 & m & $m$ & H & L & r & -1 & n & $N$ & + & $N$ & 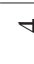 & & 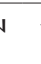 & $r$ & $\rightarrow \quad 0$ \\
\hline sse[j uుә & $r \quad m$ & $\infty$ & $-r$ & $N$ & $N$ & H & & & 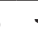 & & + & t & $m$ & N & & & $\mathrm{s}$ & N & $-r$ \\
\hline dnox: 리!S & $+\wedge$ & $\infty$ & $m$ & $\overrightarrow{6}$ & $m$ & $m$ & & & & & 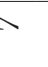 & $\ltimes$ & ت̆ & $\Lambda$ & & & 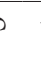 & - & $m \quad m$ \\
\hline 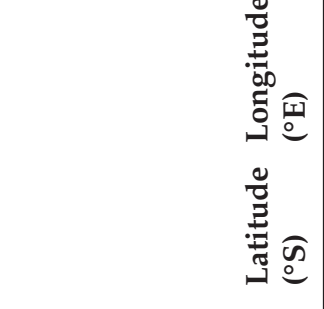 & 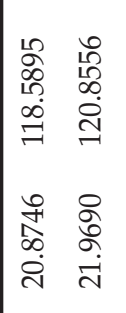 & 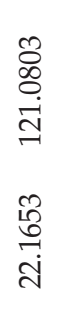 & 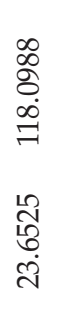 & 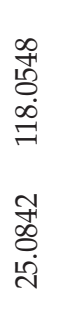 & 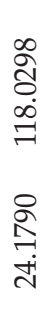 & 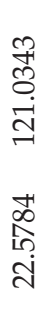 & 6 & 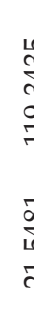 & 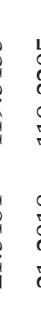 & & t. & 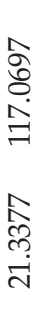 & $\begin{array}{l}\text { مू } \\
\text { ô. } \\
\dot{\sim}\end{array}$ & 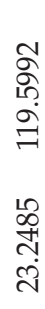 & 6 & & fi & 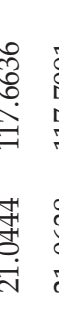 & 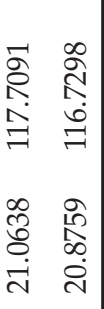 \\
\hline 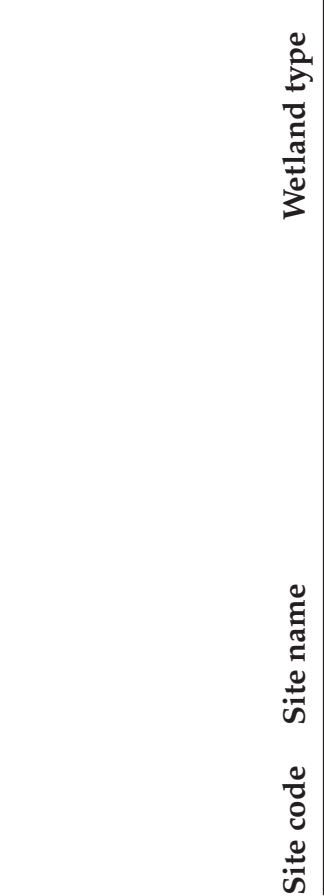 & 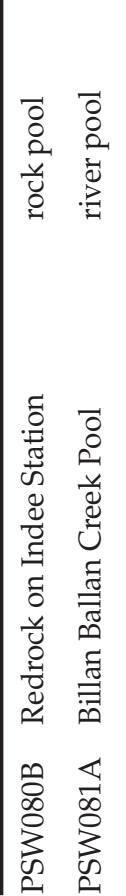 & 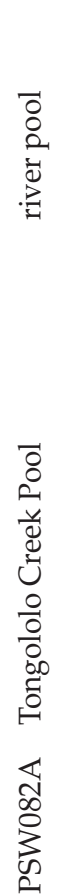 & 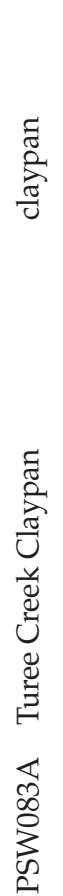 & $\begin{array}{l} \\
\overline{0} \\
0 \\
0 \\
0 \\
0 \\
5 \\
0 \\
0 \\
00 \\
0 \\
0\end{array}$ & 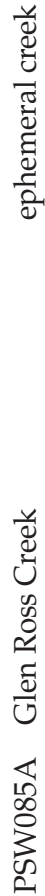 & 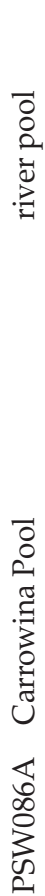 & 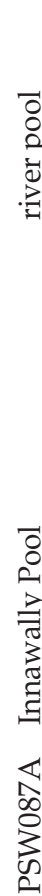 & & & & 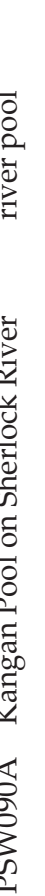 & 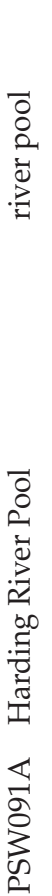 & 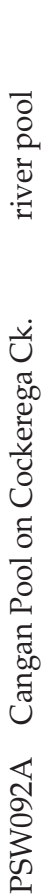 & 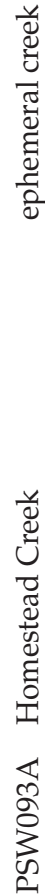 & & & 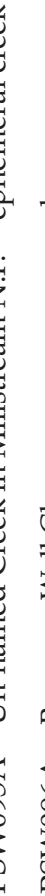 & 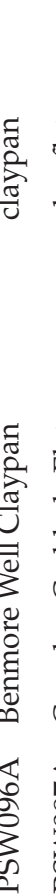 & 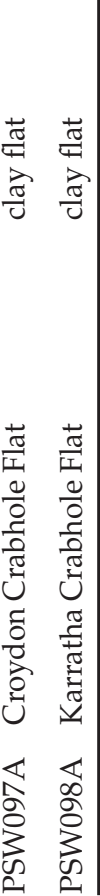 \\
\hline
\end{tabular}




\begin{tabular}{|c|c|c|c|c|c|c|c|c|c|c|c|c|c|c|c|c|c|}
\hline 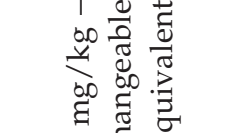 & 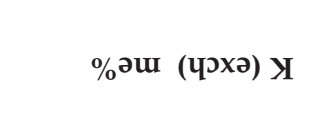 & 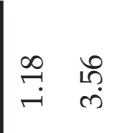 & ले & $\stackrel{\mathcal{F}}{\underset{+}{+}}$ & \llcorner & $\begin{array}{l}\text { Oे } \\
\text { ì }\end{array}$ & $\begin{array}{l}\text { ô } \\
\text { i }\end{array}$ & $\begin{array}{l}\text { Oे } \\
\text { i }\end{array}$ & ôे & 贵 & 今. & $\stackrel{\Upsilon}{\sim}$ & $\begin{array}{l}0 \\
0 \\
0\end{array}$ & in & g. & $\stackrel{\circ}{r}$ & $\stackrel{\bullet}{\rightarrow}$ \\
\hline 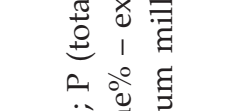 & $\%$ əuU (ЧगХə) $\mathbf{e}_{\mathbf{N}}$ & 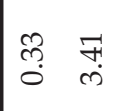 & तิ & $\stackrel{\text { ț }}{-}$ & है & 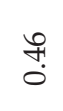 & 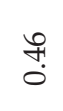 & ర్రి & $\stackrel{n}{\sim}$ & F্ & สิ & बे & $\stackrel{\hat{O}}{i}$ & 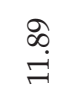 & $\stackrel{\infty}{\stackrel{\sim}{\sim}}$ & 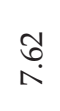 & $\stackrel{\infty}{\stackrel{0}{0}}$ \\
\hline 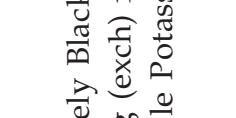 & \%əu (Чगхә) 8ి & 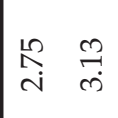 & $\stackrel{7}{i}$ & $\stackrel{\tilde{m}}{\tilde{m}}$ & 菅 & $\underset{+}{\stackrel{+}{0}}$ & $\underset{+}{\stackrel{+}{0}}$ & f & gे & $\stackrel{H}{F}$ & ભે & 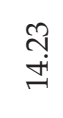 & $\stackrel{0}{\pi}$ & 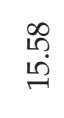 & $\stackrel{\vec{H}}{\text { ம் }}$ & $\hat{\sigma}$ & 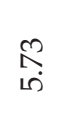 \\
\hline 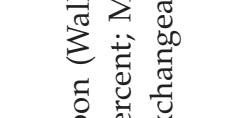 & \%әuи (чगхә) еЈ & 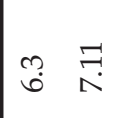 & 章 & 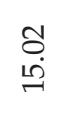 & స్తి & $\begin{array}{l}\stackrel{0}{\circ} \\
\stackrel{1}{\leftarrow}\end{array}$ & $\begin{array}{l}\stackrel{0}{\circ} \\
\stackrel{1}{\circ}\end{array}$ & $\stackrel{\infty}{\stackrel{0}{9}}$ & $\underset{7}{\stackrel{7}{+}}$ & 命 & f & $\begin{array}{l}\stackrel{8}{1} \\
\text { in }\end{array}$ & $\begin{array}{l}\infty \\
+\infty \\
+\end{array}$ & $\vec{I}$ & $\stackrel{\mathbb{N}}{N}$ & $\stackrel{\hat{n}}{=}$ & 苫 \\
\hline 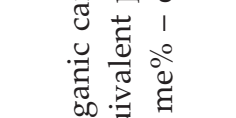 & 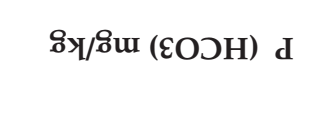 & ட & $\ddot{N}$ & $\stackrel{2}{7}$ & $\infty$ & הి & הి & เ゙ & 6 & $\infty$ & เn & $\infty$ & N & $a$ & $H$ & $m$ & $a$ \\
\hline 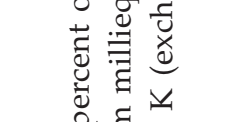 & 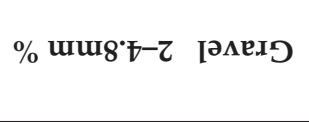 & 00 & 0 & 0 & $\circ$ & $\vec{\infty}$ & $\stackrel{\infty}{\infty}$ & o & $\stackrel{\sim}{2}$ & $\stackrel{\sigma}{\infty}$ & $\ddot{\infty}$ & Ln & $\stackrel{+}{6}$ & o & $\stackrel{+}{+}$ & 0 & 。 \\
\hline 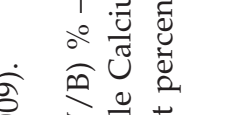 & 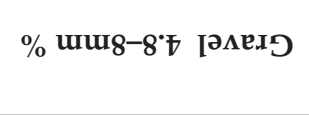 & 00 & 0 & 0 & $\circ$ & $\stackrel{m}{\rightarrow}$ & $\stackrel{m}{\stackrel{\sim}{~}}$ & 0 & 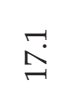 & తे & m. & $\ddot{8}$ & 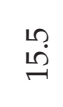 & 0 & 占 & 0 & o \\
\hline 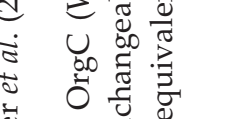 & 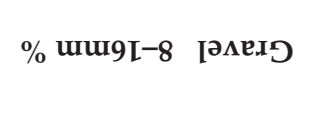 & 0 & 0 & 0 & 0 & $\stackrel{\infty}{\sigma}$ & $\stackrel{\infty}{\infty}$ & o & 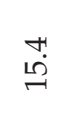 & $\stackrel{\sim}{\varrho}$ & $\begin{array}{l}\infty \\
\infty\end{array}$ & 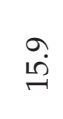 & $\stackrel{\text { Lे }}{\stackrel{9}{7}}$ & o & $\stackrel{N}{N}$ & ○ & 0 \\
\hline 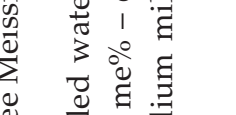 & 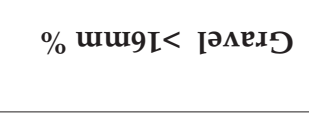 & 00 & 0 & 0 & $\circ$ & $\dot{m}$ & $\dot{m}$ & 0 & 움 & $\stackrel{0}{\circ}$ & $\stackrel{m}{+}$ & $\vec{\exists}$ & $\underset{+}{+}$ & 0 & $a$ & 0 & o \\
\hline 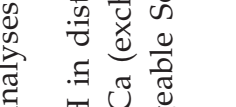 & $\%$ Ke IJ & $\stackrel{n}{m}$ & $\stackrel{n}{\stackrel{n}{N}}$ & $\begin{array}{l}\stackrel{L}{10} \\
\infty \\
\infty\end{array}$ & $\stackrel{\text { L̊ }}{\stackrel{9}{\sigma}}$ & in & in & 구 & 6 & $\stackrel{L}{\infty}$ & $H$ & $\mathbb{F}$ & $\stackrel{20}{\rightarrow}$ & $\stackrel{20}{\longrightarrow}$ & $\mathbb{H}$ & $\infty$ & F \\
\hline 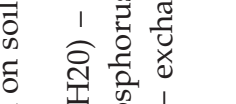 & $\%$ H!S & ๆ & $\begin{array}{l}\stackrel{10}{\circ} \\
\stackrel{2}{n}\end{array}$ & $\begin{array}{l}\stackrel{2}{2} \\
\text { ले }\end{array}$ & $\vec{m}$ & $\stackrel{\text { Ln }}{\stackrel{2}{\sigma}}$ & เి & ส & $\stackrel{\text { L̊ }}{\stackrel{0}{9}}$ & $\infty$ & $\stackrel{L}{L}$ & $\stackrel{\llcorner 0}{=}$ & $\infty$ & ¿̊. & $\stackrel{\llcorner}{=}$ & $\triangleq$ & নे \\
\hline 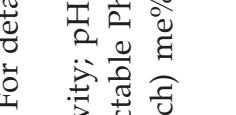 & $\%$ pues & $\begin{array}{ll}\text { மூ } \\
\text { மீं }\end{array}$ & 3 & Fे & டீ: & 埅 & F & i & $\begin{array}{l}\stackrel{\mathscr{D}}{\infty} \\
\stackrel{\infty}{\infty}\end{array}$ & $\begin{array}{l}\stackrel{L}{\infty} \\
\infty\end{array}$ & \&̊ & $\stackrel{\operatorname{Ln}}{\mathbb{H}}$ & $\hat{N}$ & $\begin{array}{l}\text { L } \\
\text { 눙 }\end{array}$ & $\stackrel{\text { Ln }}{+}$ & $\mathfrak{R}$ & $\ddot{N}$ \\
\hline 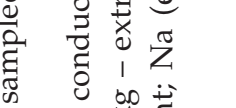 & 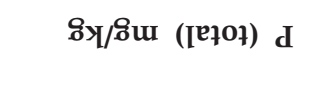 & 융 \& & R & 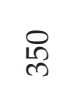 & 원 & $\underset{\infty}{\infty}$ & 文 & గ్రి & 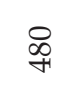 & ลิ & $\stackrel{2}{7}$ & สิ & $\stackrel{?}{7}$ & ฉి & ลิ & $\stackrel{0}{\Rightarrow}$ & 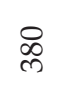 \\
\hline 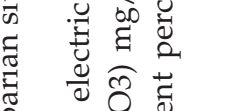 & \% (q/M) כ8ి.xO & 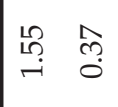 & กิ & भे & 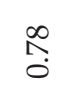 & g. & ò & - & 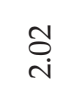 & ?̊ & $\stackrel{8}{\circ}$ & $\begin{array}{l}\infty \\
\stackrel{\infty}{0}\end{array}$ & F্ & $\stackrel{\wedge}{\oplus}$ & 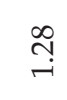 & $\stackrel{\text { S̆ }}{-}$ & $\stackrel{\stackrel{H}{\rightarrow}}{\rightarrow}$ \\
\hline 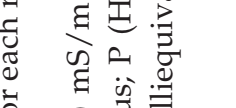 & $(O Z H) H^{d}$ & 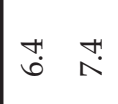 & $\stackrel{\Im}{\wedge}$ & $\stackrel{\curvearrowright}{\wedge}$ & $\underset{\infty}{\infty}$ & $\stackrel{10}{0}$ & เి & ?ु & $\stackrel{m}{\infty}$ & $\stackrel{+}{\infty}$ & $\stackrel{0}{\infty}$ & $\stackrel{+}{+}$ & $\stackrel{\infty}{\infty}$ & $\stackrel{\infty}{\infty}$ & $\stackrel{\infty}{\infty}$ & $\stackrel{\infty}{\infty}$ & ชై \\
\hline 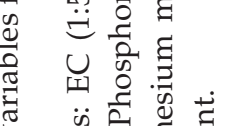 & $\begin{array}{l}u / S u \\
\text { (G:I) } \partial \exists\end{array}$ & $\infty \stackrel{9}{\stackrel{9}{二}}$ & $\stackrel{8}{\stackrel{2}{n}}$ & ลั & $\stackrel{R}{\Sigma}$ & $\infty$ & $\infty$ & $\exists$ & $R$ & 2 & 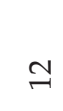 & ఫ્సి & $\infty$ & 각 & $\stackrel{10}{\infty}$ & ศิ & เn \\
\hline & 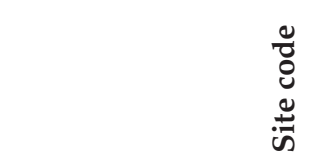 & 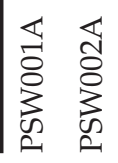 & 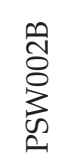 & 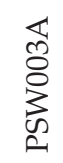 & $\begin{array}{l}\infty \\
\varnothing_{0} \\
\sum_{0}\end{array}$ & \begin{tabular}{l}
$\varangle$ \\
\multirow{2}{0}{} \\
$\sum_{0}$
\end{tabular} & 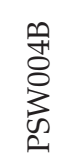 & $\begin{array}{l}\varangle \\
\text { 号 } \\
0 \\
0 \\
0\end{array}$ & $\begin{array}{l}\varangle \\
\vdots \\
0 \\
\vdots \\
0\end{array}$ & $\begin{array}{l}\mathbb{4} \\
\hat{0} \\
\vdots \\
0 \\
0\end{array}$ & $\begin{array}{l}\varangle \\
0 \\
0 \\
0 \\
\infty \\
0\end{array}$ & 点 & $\begin{array}{l}\mathbb{S} \\
0 \\
0 \\
0 \\
0\end{array}$ & $\begin{array}{l}\sharp \\
\exists \\
0 \\
0 \\
0\end{array}$ & \begin{tabular}{l}
$\mathbb{3}$ \\
\multirow{3}{0}{} \\
$\sum_{0}$
\end{tabular} & $\begin{array}{l}\overleftrightarrow{1} \\
\stackrel{3}{0} \\
\sum_{0}^{2} \\
0\end{array}$ & 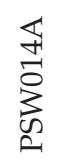 \\
\hline
\end{tabular}




\begin{tabular}{|c|c|c|c|c|c|c|c|c|c|c|c|c|c|c|c|c|c|c|c|c|c|c|c|}
\hline \%əuు (Чગхә) \્ર & $\stackrel{\infty}{\sim}$ & f & ปั & $\stackrel{\text { I }}{+\infty}$ & 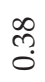 & & & $?$ & 5 & F & ?ִ & 0 & חִ & $\frac{2}{2}$ & & & 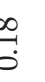 & & $\stackrel{\ln }{\circ}$ & $\stackrel{\text { fे }}{\text { r }}$ & 亦 & 7ั่ & \begin{tabular}{l}
$\stackrel{0}{\infty}$ \\
\hdashline
\end{tabular} \\
\hline \%əuा (чगхә) e $N$ & $\underset{ल}{\vec{\rho}}$ & - & $\stackrel{\widehat{\sigma}}{-}$ & $\begin{array}{l}\stackrel{1}{0} \\
0\end{array}$ & ֻొن & & & نـ & $\overbrace{i}^{2}$ & ָ̃ & $\stackrel{+}{\stackrel{+}{\leftarrow}}$ & ن & $\begin{array}{c}\infty \\
\infty \\
\infty \\
\infty\end{array}$ & s & & & 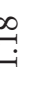 & 定 & 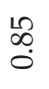 & $\stackrel{\sim}{\sim}$ & $\stackrel{+}{\stackrel{H}{0}}$ & $\stackrel{\text { ִָ }}{0}$ & مُ \\
\hline 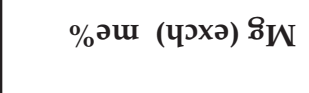 & $\begin{array}{l}\infty \\
\stackrel{0}{0}\end{array}$ & $\stackrel{\infty}{0}$ & $\stackrel{\sigma}{\rightarrow}$ & $\underset{\text { ¿े }}{\text { i }}$ & 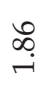 & in & & 渶 & tit & $\overrightarrow{+}$ & $\begin{array}{l}\text { రి } \\
\text { ம் }\end{array}$ & ఝु. & 赵 & $q^{g}$ & & & $\overrightarrow{\mathrm{N}}$ & : & તิ & \begin{tabular}{l}
0 \\
0 \\
0 \\
\hdashline
\end{tabular} & તુ & 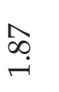 & 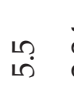 \\
\hline \%əü (Чगхә) е & 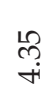 & กิ & ఝु & ले & 7 & ภิ & & ما & ל้ & ڤ్ & 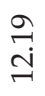 & 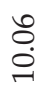 & 站 & 9 & & & $\overrightarrow{\mathrm{i}}$ & సิ & $\vec{N}$ & 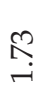 & $\begin{array}{l}\infty \\
\stackrel{\infty}{0}\end{array}$ & & $\underset{+}{\stackrel{H}{*}}$ \\
\hline 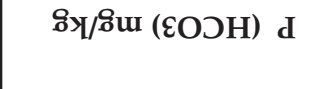 & $\sigma$ & $m$ & H & $\mathcal{I}$ & $\stackrel{9}{\rightarrow}$ & c & & م & A & $\stackrel{6}{-}$ & $\approx$ & $a$ & $m$ & 5 & & & م & c) & $\stackrel{2}{\sim}$ & $\stackrel{m}{\rightarrow}$ & 几 & $\stackrel{\sim}{\sim}$ & $\llcorner n$ \\
\hline 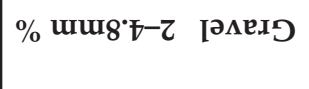 & ○ & $\hat{ِ}$ & 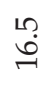 & $\stackrel{\text { N }}{\exists}$ & 0 & $\stackrel{\leftarrow}{2}$ & & b & $\sigma$ & o & ○ & ले & ה & c & & $b$ & הี & 0 & 0 & o & ○ & 0 & ○ \\
\hline 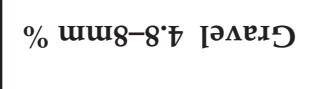 & 0 & $\stackrel{\infty}{\wedge}$ & $\stackrel{\infty}{6}$ & $\stackrel{a}{\grave{\omega}}$ & 0 & & & b & $\stackrel{2}{\sim}$ & 0 & 0 & 2 & I & 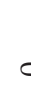 & & $b$ & ça & 0 & 0 & 0 & 0 & 0 & 0 \\
\hline 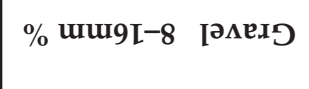 & 0 & $\stackrel{0}{\stackrel{0}{0}}$ & $\begin{array}{l}\stackrel{0}{\dot{\oplus}} \\
\stackrel{\leftrightarrow}{2}\end{array}$ & $\hat{ت}$ & 0 & s & & o & \pm & $\circ$ & ○ & i & t. & c & & & "i. & 0 & o & o & 0 & 0 & ○ \\
\hline 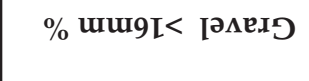 & 0 & 点 & تॄ. & $a$ & 0 & & & o & $\sigma_{\infty}$ & 0 & 0 & 6 & 6 & 0 & & $b$ & S. & 0 & 0 & 0 & 0 & 0 & 0 \\
\hline$\%$ Ke IJ & $\stackrel{1}{\infty}$ & H & $\wedge$ & $\underset{\sim}{\sim}$ & $\wedge$ & $m$ & & $n^{n}$ & เ & ڤृ & 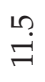 & 우 & L & $c$ & & $?_{i}^{+}$ & + & ले & نُ & $\stackrel{m}{\rightarrow}$ & + & $\wedge$ & ले \\
\hline$\%$ HI!S & $\mathbb{H}$ & $\infty$ & $\stackrel{\llcorner}{\llcorner}$ & $\wedge$ & 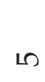 & L & & 疍 & 6 & $\approx$ & $\exists$ & 冓 & $\Lambda$ & (c) & & $?$ & הִ & m & 욱 & 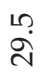 & N & $\wedge$ & $a$ \\
\hline$\%$ pues & $\stackrel{\text { ?ִ }}{\stackrel{1}{人}}$ & $\infty$ & $\begin{array}{l}\stackrel{\llcorner}{\infty} \\
\stackrel{\infty}{\infty}\end{array}$ & $\bar{\infty}$ & $\infty$ & $\sigma$ & & m & $\infty$ & $\frac{\operatorname{l}_{\infty}}{\dot{\infty}^{\prime}}$ & 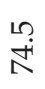 & $\stackrel{\infty}{\infty}$ & $\infty$ & 8 & & R & תֵ & సิ & 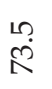 & 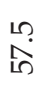 & హ゙ & $\infty$ & กิ \\
\hline 8צ//8u ([एłoł) d & கి & $\stackrel{\sim}{\infty}$ & 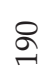 & 员 & 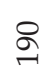 & $£$ & & : & हे & 류 & $\stackrel{\circ}{\vec{m}}$ & $\begin{array}{l}\infty \\
\infty \\
\infty\end{array}$ & ङे & 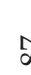 & & b & 을 & $\begin{array}{l}\infty \\
\infty \\
\infty\end{array}$ & 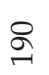 & 呙 & n & 원 & ભి \\
\hline 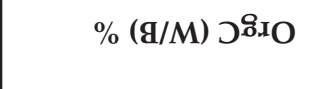 & گิ & - & $\stackrel{\text { L }}{\circ}$ & 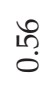 & 苞 & ल & & . & 离 & & ন্ন & 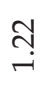 & $\begin{array}{c}\infty \\
\infty \\
0 \\
0\end{array}$ & 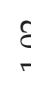 & & هُ & "ְ. & - & ָ̊ & $\stackrel{\overbrace{}}{ت}$ & 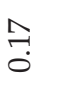 & $\begin{array}{l}\vec{\infty} \\
0 \\
0\end{array}$ & $\stackrel{H}{\stackrel{H}{0}}$ \\
\hline$(\mathrm{OZH}) \mathrm{H}^{\mathrm{d}}$ & $a$ & $\stackrel{+}{\infty}$ & $a$ & $\stackrel{+}{\infty}$ & $\infty$ & $\sigma$ & & ơ & مْ & $a$ & $\infty$ & $\infty$ & 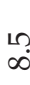 & 5 & & 。 & r. & فْ & $\stackrel{\text { म }}{\circ}$ & $\stackrel{\rho}{\infty}$ & $\stackrel{+!}{\Lambda}$ & જ̆ & $\stackrel{3}{N}$ \\
\hline $\begin{array}{c}u / S u \\
(\mathrm{G}: \mathrm{I}) \quad \mathrm{J}\end{array}$ & $\hat{6}$ & 요 & ले & $\stackrel{\infty}{\infty}$ & $\ddot{\sim}$ & $\underline{a}$ & & $m$ & = & ले & $\underset{0}{8}$ & \& & f & to & & H & ते & 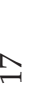 & 우 & 号 & H & in & $\wedge$ \\
\hline 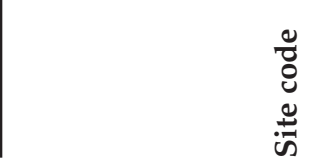 & 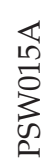 & 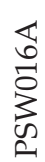 & 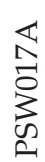 & 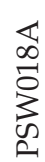 & 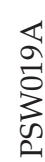 & 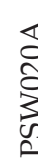 & & 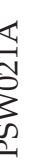 & 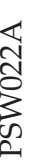 & 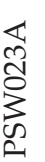 & 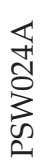 & 岕 & 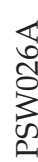 & 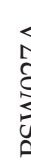 & & & 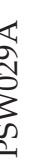 & 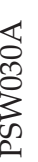 & 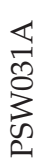 & 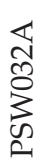 & 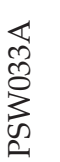 & 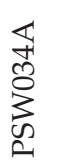 & 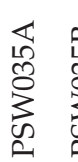 \\
\hline
\end{tabular}




\begin{tabular}{|c|c|c|c|c|c|c|c|c|c|c|c|c|c|c|c|c|c|c|c|c|c|c|c|}
\hline \%əuा (Чगхә) પ્ર & 皿 & -3 & & & & à & i & 今. & $\stackrel{F}{ت}$ & ? & $\bar{c}$ & & ț & & ले & ฟู & ڤె. & $\stackrel{0}{\circ}$ & $\stackrel{\substack{0 \\
0}}{\circ}$ & भे & ?ִ & $\stackrel{\sim}{\sim}$ & ले \\
\hline \%əu (чगхә) $\mathbf{e}_{\mathbf{N}}$ & तิ & $\stackrel{\infty}{\circ}$ & Lᄂ & & & I্ & 䞒 & $\stackrel{0}{-}$ & $\stackrel{8}{0}$ & ई & 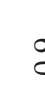 & & $\begin{array}{l}\text { va } \\
\text { m. }\end{array}$ & ô. & m్ & $\stackrel{\text { ֻึ }}{0}$ & ָึ & - & $\stackrel{\infty}{N}$ & $\stackrel{\infty}{0}$ & $\underset{\text { Ș }}{-}$ & के & م \\
\hline \%əu (Чәхә) $8 \mathrm{~W}$ & तิ & $\stackrel{\stackrel{L}{n}}{0}$ & 5 & & 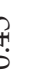 & $\underset{\text { F }}{\stackrel{F}{i}}$ & $\stackrel{+}{\stackrel{+}{r}}$ & $\begin{array}{l}\stackrel{\curvearrowright}{n} \\
\text { n. }\end{array}$ & : & $\stackrel{4}{9}$ & 7 & & ?ִ & 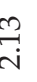 & $\stackrel{\operatorname{Ln}}{\mathrm{i}}$ & نمٌ & مي & 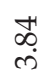 & $\stackrel{\text { ?ִ }}{\rightarrow}$ & ભి & ָ̃ & 꼬 & ఝో \\
\hline \%әи (чЈхә) вЈ & $\begin{array}{l}-1 \\
\sigma\end{array}$ & : & 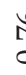 & & $\stackrel{f}{s}$ & 䒜 & గก & : & 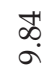 & $\bar{c}$ & ริ & & לְ & $\tilde{S}_{+}^{2}$ & 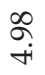 & $\widehat{\wedge}$ & مٌ & 点 & $\stackrel{\text { ڤ }}{\text { in }}$ & ठै. & $\underset{⿱ 乛}{\stackrel{N}{N}}$ & $\vec{b}$ & تु \\
\hline 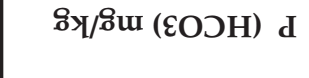 & $\exists$ & 6 & L & & th & $\approx$ & 9 & $\stackrel{m}{\sim}$ & $\Lambda$ & 于 & $c$ & & 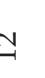 & $\sigma$ & 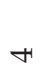 & $a$ & $F$ & in & H & 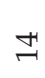 & $\approx$ & $\stackrel{10}{\longrightarrow}$ & 요 \\
\hline 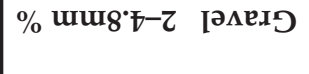 & 0 & 0 & 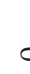 & & & 0 & 0 & 0 & 0 & 0 & c & & th & e & 0 & $\stackrel{\infty}{+}$ & o & o & o & $\stackrel{\text { I }}{\text { I }}$ & o & 0 & 0 \\
\hline 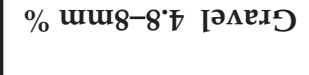 & 0 & 0 & c & & b & 0 & 0 & 0 & 0 & c & c & & לֶ. & 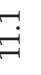 & 0 & $\vec{m}$ & o & 0 & o & $\stackrel{H}{二}$ & 0 & ○ & 0 \\
\hline 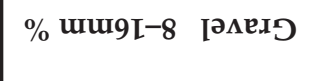 & 0 & 0 & c & & D & 0 & 0 & 0 & 0 & c & c & & $\stackrel{?}{?}$ & 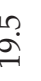 & o & $\stackrel{\infty}{\oplus}$ & o & 0 & o & 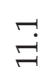 & o & o & 0 \\
\hline 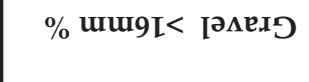 & 0 & 0 & c & & b & 0 & 0 & 0 & 0 & c & c & & זُ+ & ה? & 0 & iे & ○ & 0 & o & $\dot{m}$ & o & o & 0 \\
\hline$\%$ Ke & 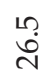 & $\mathrm{s}$ & s & & th & 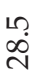 & $\vec{m}$ & के & $\infty$ & 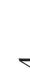 & Lᄂ & & ְُ & 6 & 욱 & $\stackrel{\llcorner}{+}$ & 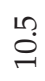 & $\stackrel{\llcorner}{\longrightarrow}$ & $\stackrel{\infty}{\sim}$ & $a$ & $a$ & $F$ & Ln: \\
\hline$\%$ HI!S & $\curvearrowright$ & $\stackrel{20}{0}$ & r & & - & $\stackrel{m}{\sim}$ & 官 & $a$ & 0 & 0 & s & & ? & קח & $F$ & $\stackrel{\llcorner}{+}$ & $a$ & $\stackrel{L ?}{\wedge}$ & ले & $\infty$ & $a$ & $\stackrel{0}{\sim}$ & $\infty$ \\
\hline$\%$ purs & $\begin{array}{l}\text { Lी } \\
\text { மீ }\end{array}$ & $\begin{array}{l}\stackrel{\llcorner}{\Lambda} \\
\stackrel{\alpha}{\sigma}\end{array}$ & 8 & & $R$ & in & in & L & $\infty$ & $\delta$ & 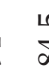 & & $\infty$ & $\infty$ & 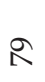 & $\sigma$ & $\begin{array}{l}\text { L } \\
\infty \\
\infty\end{array}$ & $\vec{\infty}$ & กิ & $\infty$ & $\infty$ & m & L \\
\hline 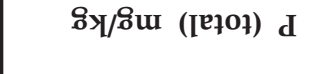 & 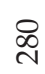 & $\infty$ & ז̊ & & $R$ & \&্ণ & 요 & & 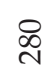 & $£$ & $\rho_{2}^{2}$ & & 导 & बे & ڤ્స & $\stackrel{8}{\circ}$ & లి & $\underset{\sim}{\sim}$ & $\alpha$ & 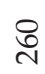 & $\stackrel{ }{*}$ & 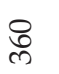 & 권 \\
\hline \% (g/M) כ. & @ం & $\stackrel{0}{\circ}$ & 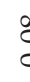 & & $\frac{0}{4}$ & సֶ. & ત્ર & F & $\stackrel{\infty}{\circ}$ & $\bar{c}$ & E & 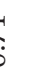 & th & हैं & مُ & $\stackrel{\sharp}{\sharp}$ & Iี & 茴 & $\stackrel{\text { LP }}{\circ}$ & @ై & f & ڤ̊ & 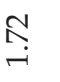 \\
\hline$(\mathrm{OZH}) \mathrm{H}^{\mathrm{d}}$ & $\stackrel{0}{0}$ & เి & r & & P & $\stackrel{m}{\wedge}$ & $\stackrel{+}{\Lambda}$ & 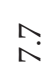 & $\infty$ & 2 & s & & ?ִ & $\underset{\infty}{+\infty}$ & $\curvearrowright$ & $\begin{array}{ll}\llcorner \\
\infty \\
\infty\end{array}$ & $\stackrel{3}{\infty}$ & $\underset{\infty}{+!}$ & $\ddot{a}$ & $\stackrel{\llcorner}{\stackrel{2}{~}}$ & $\infty$ & 6 & $\underset{\infty}{\infty}$ \\
\hline $\begin{array}{c}\mathbf{w} / \mathbf{S u} \\
(\mathrm{g}: \mathrm{L}) \quad \mathrm{J}\end{array}$ & 6 & 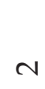 & 0 & & o & ले & $\infty$ & $\approx$ & ৪্ল & ปุ & ๙ & $\frac{1}{1}$ & D্ & o & in & $\vec{\sim}$ & त) & मे & $\stackrel{\sim}{\sim}$ & $\stackrel{\stackrel{2}{N}}{\sim}$ & ス & $\infty$ & F \\
\hline 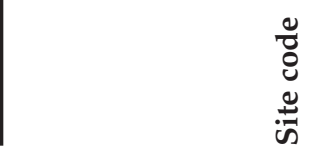 & 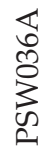 & 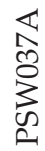 & d & & 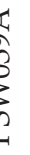 & 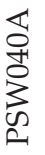 & 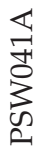 & 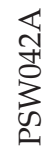 & 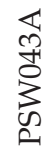 & 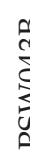 & 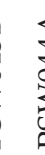 & & 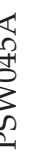 & 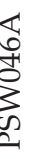 & 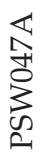 & 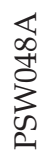 & $\begin{array}{l}\overleftarrow{\sigma} \\
\text { †े } \\
3 \\
\text { के }\end{array}$ & 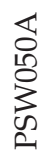 & 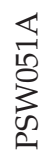 & 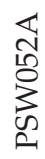 & 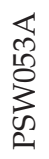 & 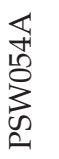 & 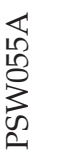 \\
\hline
\end{tabular}




\begin{tabular}{|c|c|c|c|c|c|c|c|c|c|c|c|c|c|c|c|c|c|c|c|c|c|c|c|c|}
\hline \%əuు (Чગхә) \્ર & 7 & 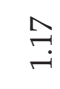 & & 占 & & & & & & & సี & חై & & & & & & ले & $\begin{array}{l}\infty \\
\stackrel{0}{0} \\
\dot{0}\end{array}$ & สิ & $\stackrel{\infty}{\rightarrow}$ & F্ & 苗 & $\begin{array}{cc}\vec{\infty} & \overrightarrow{0} \\
0 & 0\end{array}$ \\
\hline \%əuा (чगхә) e $N$ & ๙ิ & กิ่ & $\infty$ & $\hat{\jmath}$ & & & & c & & $\dot{3}$ & ले & 管 & . & $\mathrm{c}$ & & & & $\stackrel{0}{\circ}$ & $\bar{m}$ & $\stackrel{\sim}{\rightarrow}$ & స్ & Iั & 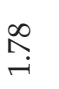 & \begin{tabular}{ll}
$\stackrel{2}{n}$ & $\stackrel{L}{0}$ \\
\hdashline & 0 \\
0 & 0
\end{tabular} \\
\hline \%əu & S. & : & I & b & & ç & 卓 & 2 & & \begin{tabular}{l}
0 \\
0 \\
0 \\
\hdashline
\end{tabular} & $\underset{ت}{ت}$ & $\stackrel{\text { L }}{\mathrm{d}}$ & लై & $\begin{array}{ll}a \\
\text { a }\end{array}$ & & & . & $\begin{array}{l}\tilde{c} \\
\dot{\theta}\end{array}$ & $\stackrel{\llcorner}{\llcorner\circ}$ & : & i⿱宀 & $\begin{array}{l}\text { Cू } \\
\text { ले }\end{array}$ & ભొ & ले సै \\
\hline \%əu (ЧગХว) вЈ & $\begin{array}{l}\infty \\
\infty \\
i\end{array}$ & 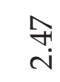 & $\begin{array}{l}c \\
\infty \\
\infty\end{array}$ & o & & ?. & לु. & 5 & & ? & مج & $\begin{array}{c}\hat{m} \\
\text { ले }\end{array}$ & ڤึ & $\frac{\partial}{q}$ & & & E & $\stackrel{\widetilde{F}}{\overrightarrow{+}}$ & นִ & $\stackrel{\text { L }}{\stackrel{\leftrightarrow}{-}}$ & $\underset{\text { 구 }}{7}$ & $\begin{array}{l}\infty \\
\infty \\
\end{array}$ & ले & $\underset{+}{\stackrel{F}{F}}$ \\
\hline 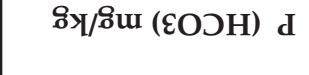 & $\omega$ & in & $\sim$ & 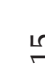 & & + & 6 & s & & $\begin{array}{c}v^{2} \\
0\end{array}$ & $n$ & H & $m$ & $\mathrm{~N}$ & & - & N & ส & $\stackrel{L}{N}$ & $\alpha$ & $\stackrel{ }{\rightarrow}$ & $\infty$ & 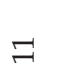 & ה \\
\hline 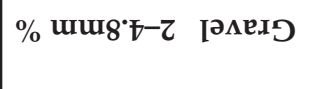 & ○ & $\vec{\sigma}$ & 0 & $c$ & & $m$ & o & $c$ & & b & 0 & $\stackrel{\circ}{-1}$ & $\bar{v}$ & $c$ & & $b$ & & 0 & 0 & 0 & o & 0 & $\stackrel{\bullet}{\stackrel{0}{\circ}}$ & 00 \\
\hline 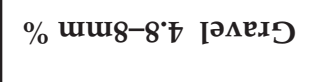 & o & $\begin{array}{l}\infty \\
\stackrel{\infty}{\rightarrow} \\
\sim\end{array}$ & o $1+1-1+1$ & $c$ & & 6 & 0 & $c$ & & b & 0 & $\mathcal{I}$ & $\stackrel{\text { I }}{~}$ & 0 & & b & $\sigma^{\circ}$ & 0 & 0 & 0 & 0 & 0 & $\begin{array}{l}\infty \\
\stackrel{\sim}{\sim}\end{array}$ & 00 \\
\hline 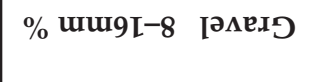 & ○ & $\hat{\mathrm{i}}$ & 0 & $c$ & & in & 0 & c & & o & 0 & ป & aे & c & & & 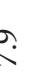 & ○ & o & o & o & 0 & $\begin{array}{l}\stackrel{+}{0} \\
\stackrel{\infty}{\rightarrow}\end{array}$ & 00 \\
\hline 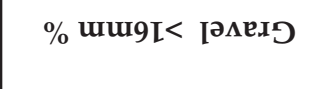 & 0 & $\stackrel{+}{\leftrightarrow}$ & c & $c$ & & $\infty$ & 0 & $c$ & & b & o & तิ & Li & $c$ & & $b$ & $m^{\circ}$ & 0 & 0 & 0 & 0 & 。 & تே. & 00 \\
\hline$\%$ Ke IJ & $\stackrel{\llcorner}{i}$ & $\stackrel{\llcorner}{\oplus}$ & $\infty$ & Lr & & ה & เి & c & & ב & $H$ & H & 6 & $\stackrel{4}{m}$ & & i & + & 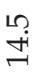 & 눙 & นึ? & के & $\wedge$ & $a$ & 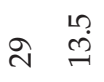 \\
\hline$\%$ HI!S & $\stackrel{L}{0}$ & $m$ & $\infty$ & $\infty$ & & קִ & $\stackrel{2}{\circ}$ & $\mathrm{a}$ & & 3 & Lִ & nृ. & in & L & & v & & 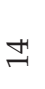 & 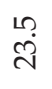 & $\stackrel{\text { L̊ }}{0}$ & $\approx$ & 管 & 6 & $\infty$ \\
\hline$\%$ pues & ล & $\begin{array}{l}\stackrel{10}{\sigma} \\
\sigma\end{array}$ & $\infty$ & $\begin{array}{l}\text { L̊ } \\
\text { a }\end{array}$ & & ๑ & \$ & 낭 & & 6 & $\begin{array}{l}\text { L? } \\
\text { \&̊ }\end{array}$ & ส่ & $\begin{array}{l}l 0 \\
\infty \\
\infty \\
\infty\end{array}$ & एँ & & ? & & $\stackrel{\text { nִ }}{\circledR}$ & $\stackrel{\llcorner}{m}$ & 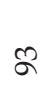 & 号 & $\begin{array}{l}\stackrel{2}{0} \\
\text { i } \\
\infty\end{array}$ & $\stackrel{10}{\infty}$ & 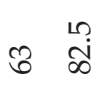 \\
\hline 8צ//8u ([एłoł) d & $\stackrel{ }{\exists}$ & 吕 & f & $\lesssim$ & & $\stackrel{2}{8}$ & 곡 & 㟶 & & D & 윽 & m. & $\stackrel{\infty}{\infty}$ & 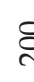 & & e & ST & ৪ & 각 & $\stackrel{\infty}{\wedge}$ & ন্ & 㖼 & 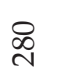 & 을 \\
\hline 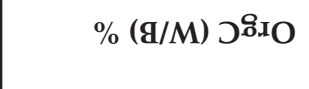 & 今े & $\stackrel{\infty}{3}$ & حે & $\hat{\jmath}$ & & ले & $\stackrel{\mathbb{H}}{\rightarrow}$ & 过 & & 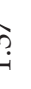 & 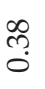 & 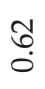 & ? & 7 & & $?$ & $\vec{\nabla}$ & 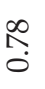 & $\stackrel{\text { If }}{\rightarrow}$ & $\stackrel{0}{\circ}$ & $\begin{array}{l}\text { స్ } \\
\text { o }\end{array}$ & 足 & 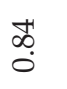 & $\begin{array}{ll}\stackrel{0}{0} & ? \\
0 & 0\end{array}$ \\
\hline$(\mathrm{OZH}) \mathrm{H}^{\mathrm{d}}$ & $\stackrel{m}{\infty}$ & $\stackrel{\pi}{\Lambda}$ & $\infty$ & a & & ָै & $\stackrel{\infty}{\infty}$ & $\alpha$ & & $\dot{0}$ & $\wedge$ & $\stackrel{+}{\infty}$ & $\infty_{\infty}^{\infty}$ & L & & ب! & $10^{\circ}$ & ชู & กี่ & สุ & $\stackrel{n}{n}$ & $\underset{\infty}{\infty}$ & $\underset{\infty}{\infty}$ & ウे \\
\hline $\begin{array}{c}u / S u \\
(\mathrm{G}: \mathrm{I}) \quad \mathrm{J}\end{array}$ & 6 & 구 & $\stackrel{\infty}{m}$ & $£$ & & $\vec{m}$ & 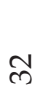 & $\stackrel{150}{7}$ & & 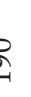 & 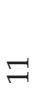 & $\stackrel{4}{4}$ & ొ & 7 & & H & m & $\stackrel{2}{\sim}$ & $a$ & 6 & $\approx$ & ஓ & ঐి & $\wedge \ddot{\sim}$ \\
\hline 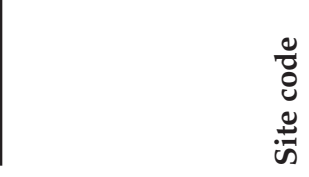 & 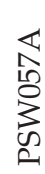 & 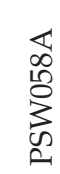 & है & $\begin{array}{l}5 \\
d \\
3 \\
0\end{array}$ & & $\frac{1}{3}$ & 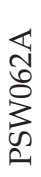 & 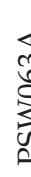 & & 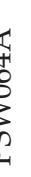 & 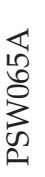 & 离 & 交 & 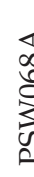 & & & $?$ & 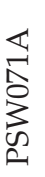 & 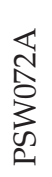 & 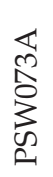 & 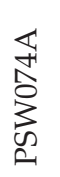 & 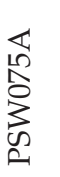 & 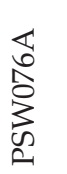 & 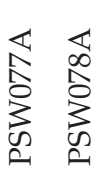 \\
\hline
\end{tabular}




\begin{tabular}{|c|c|c|c|c|c|c|c|c|c|c|c|c|c|c|c|c|c|c|c|c|c|}
\hline \%əü (чગхә) પ્ર & 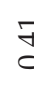 & & ָึ & $\stackrel{\mathrm{S}}{\mathrm{\delta}}$ & & & ?ִ & & חֶ. & نٌْ & $\stackrel{0}{0}$ & 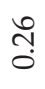 & $\begin{array}{l}\text { İ } \\
\text {. }\end{array}$ & $\stackrel{\mathbb{N}}{0}$ & $\stackrel{m}{0}$ & 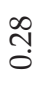 & તิ & חֶ. & જิ & 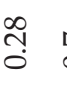 & $\stackrel{8}{8}$ \\
\hline \%əu (чगхә) $\mathbf{e}_{\mathbf{N}}$ & $\stackrel{4}{2}$ & $\stackrel{\circ}{\circ}$ & గิ & $\bar{\sigma}$ & ले & & s. & in & $\begin{array}{l}\text { ț } \\
\text { i }\end{array}$ & 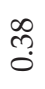 & : & 犬̂̀ & $\vec{\oplus}$ & $\stackrel{8}{\circ}$ & 㤐 & ते & กั & $\stackrel{+}{5}$ & તิ & $\begin{array}{l}8 \\
0 \\
0\end{array}$ & $\begin{array}{l}\infty \\
b \\
b\end{array}$ \\
\hline \%әши (чวхә) & ले & I & $\widehat{\curvearrowright}$ & तु & $\frac{1}{4}$ & & fo & $\begin{array}{l}+ \\
\dot{0} \\
\dot{0}\end{array}$ & $\stackrel{\infty}{\stackrel{\sim}{\rightleftarrows}}$ & $\underset{+}{ָ}$ & $\underset{ָ}{+}$ & $\grave{0}$ & $\underset{\infty}{\hat{\infty}}$ & $\stackrel{\stackrel{0}{\sim}}{\mathrm{i}}$ & กิ? & $\stackrel{\text { L }}{\rightarrow}$ & $\stackrel{\infty}{\stackrel{\infty}{-}}$ & $\underset{\vec{i}}{\stackrel{i}{*}}$ & तु & ס̊ & 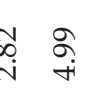 \\
\hline \%əu (чगхә) в & वृ & $\stackrel{\text { H }}{-}$ & $\stackrel{\overbrace{}}{ت}$ & $\underset{+}{\mathbb{T}}$ & $\bar{\alpha}$ & & : & $\stackrel{0}{\circ}$ & $\stackrel{g}{q}$ & $\overparen{\widehat{~}}$ & $\stackrel{\vec{े}}{\ddot{m}}$ & iे & $\stackrel{\text { I }}{+}$ & 로. & مึ & $\begin{array}{l}\vec{\infty} \\
\dot{\infty}\end{array}$ & $\begin{array}{l}\text { م⿱⺈ } \\
\text { Ln }\end{array}$ & हn & 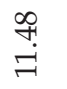 & مُ & 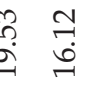 \\
\hline sy//su $(\varepsilon O \supset H) d$ & $\stackrel{\infty}{2}$ & m & ๙ & নे & 7 & & 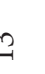 & $\Lambda$ & $\exists$ & 우 & $m$ & $\Lambda$ & $\infty$ & 6 & 6 & in & 6 & H & H & $\infty$ & $\infty \quad n$ \\
\hline$\%$ uU⿴囗十 & $c$ & $\stackrel{+}{\circ}$ & $\stackrel{?}{\Omega}$ & 0 & $\infty$ & & b & $\begin{array}{l}\stackrel{0}{0} \\
\stackrel{0}{-}\end{array}$ & $\stackrel{\sim}{\stackrel{\sim}{\sim}}$ & 0 & ○ & $\begin{array}{l}\infty \\
\stackrel{-}{-}\end{array}$ & ○ & $\stackrel{\curvearrowright}{+}$ & $\stackrel{2}{\sim}$ & ָे. & $\begin{array}{l}\stackrel{\leftrightarrow}{\sim} \\
\vec{\lambda}\end{array}$ & बें & $\circ$ & 0 & 00 \\
\hline 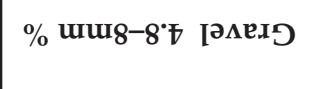 & $c$ & $\overparen{\sim}$ & $\stackrel{\sharp}{\circ}$ & o & ģ & & b & $\stackrel{\curvearrowright}{\wedge}$ & 0 & o & 0 & تே. & 0 & H & மे & 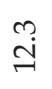 & 占 & $\stackrel{\infty}{\exists}$ & ○ & $\circ$ & 00 \\
\hline 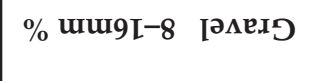 & $c$ & $\widetilde{n}$ & $\mathbb{E}$ & 0 & 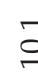 & & o & $\vec{\infty}$ & $\stackrel{0}{\sigma}$ & 0 & 0 & $\begin{array}{l}\text { ते } \\
\text { ஸे }\end{array}$ & ○ & तै & $\stackrel{\text { L }}{\stackrel{0}{\circ}}$ & $\stackrel{0}{\underset{+}{+}}$ & Lొ & $\overrightarrow{5}$ & 0 & 0 & 00 \\
\hline 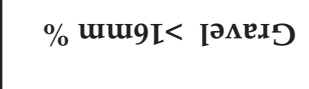 & $c$ & $\stackrel{+}{i}$ & $\vec{\AA}$ & o & $\underset{\alpha}{\alpha}$ & & b & لृ & ชู & o & ○ & $\begin{array}{l}\infty \\
\stackrel{+}{+}\end{array}$ & ○ & $\stackrel{\circ}{\circ}$ & $\begin{array}{l}\stackrel{\infty}{\circ} \\
\stackrel{\rho}{\sim}\end{array}$ & $\stackrel{+}{\therefore}$ & $\stackrel{\infty}{=}$ & $\ddot{\infty}$ & 0 & ○ & 00 \\
\hline$\%$ Ke & $\stackrel{L}{\infty}$ & $\infty$ & $\Lambda$ & 으 & g & & ?: & $\stackrel{0}{\sim}$ & $a$ & $\stackrel{n}{\mathbb{U}}$ & เి & $\stackrel{n}{+\infty}$ & in & $\stackrel{\ln }{+\rightarrow}$ & $\stackrel{\ln }{\stackrel{\ln }{9}}$ & $\stackrel{\operatorname{Ln}}{+}$ & H & $a$ & 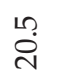 & 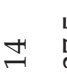 & 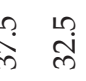 \\
\hline$\%$ HI!S & 6 & $\stackrel{10}{\varrho}$ & $\Lambda$ & $\stackrel{M}{\sim}$ & 0 & & م & $\stackrel{M}{\sim}$ & $\Lambda$ & 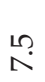 & H & H & $\infty$ & $\stackrel{10}{\infty}$ & $\approx$ & $m$ & $\stackrel{\leftrightarrow}{\infty}$ & $\stackrel{n}{0}$ & $\stackrel{10}{2}$ & นִ & $\stackrel{\leftrightarrow}{\circ}$ \\
\hline$\%$ pues & 它 & $\stackrel{\text { ?ִ }}{\infty}$ & $\infty$ & 太 & N & & ?: & 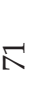 & 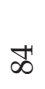 & $\infty$ & 号 & $\frac{\operatorname{La}}{\dot{\alpha}}$ & $\omega_{\infty}$ & $\alpha$ & $\stackrel{\text { L? }}{\stackrel{N}{\Lambda}}$ & $\begin{array}{l}\text { L } \\
\text { S }\end{array}$ & $\begin{array}{l}\text { L } \\
\text { S }\end{array}$ & $\begin{array}{l}\stackrel{1}{0} \\
\stackrel{\infty}{\infty}\end{array}$ & 8 & مْ & 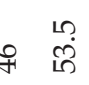 \\
\hline 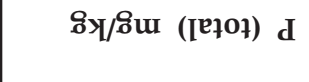 & ले & ণ్తి & $\stackrel{\infty}{\infty}$ & ஓ্ল & वृ & & 2 & ¿ & ळे & নे & సิ & $\stackrel{\infty}{\infty}$ & สิ & 왁 & ले & 呙 & $\stackrel{\text { I }}{N}$ & ৪্র & ลิ & ळ & జి \\
\hline 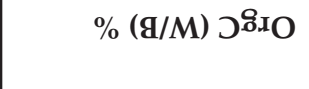 & a & $\stackrel{\infty}{+}$ & Iี & 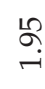 & ç & & पै & مُ & $\stackrel{\text { L }}{\circ}$ & $\stackrel{m}{\rightarrow}$ & 年 & 莆 & $\stackrel{t}{\leftrightarrow}$ & 芯 & $\stackrel{N}{\stackrel{N}{-}}$ & ㅊ. & ले & $\stackrel{\circ}{\circ}$ & $\stackrel{\vec{\sigma}}{\rightarrow}$ & तु & 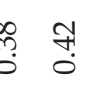 \\
\hline$(\mathrm{OZH}) \mathrm{H}^{\mathrm{d}}$ & छु & ○ & ?3. & a & $\mathrm{N}$ & & 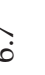 & $\stackrel{\circ}{\circ}$ & $\stackrel{\circ}{\sim}$ & $\stackrel{\curvearrowright}{\curvearrowright}$ & $\stackrel{\circ}{\circ}$ & ॠ̆ & $\underset{\infty}{\infty}$ & $\underset{\infty}{0}$ & 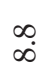 & $\stackrel{0}{\circ}$ & $\operatorname{Ln}_{\infty}^{\infty}$ & मु & $\infty$ & ف: & $\infty \quad \begin{array}{l}0 \\
\infty\end{array}$ \\
\hline $\begin{array}{c}u / g u \\
(\mathrm{~g}: \mathrm{I}) \quad \mathrm{J}\end{array}$ & 6 & H & ㄱ. & $\stackrel{\ominus}{\exists}$ & 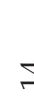 & & $=$ & छூ & $\stackrel{8}{-}$ & $\stackrel{M}{M}$ & 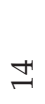 & ถి & ஓ & 9 & హ゙ & F & $\infty$ & เn & $\Rightarrow$ & N & $\approx$ \\
\hline 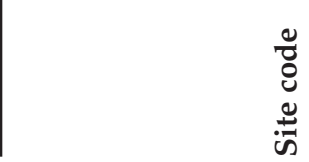 & ڤ. & $\begin{array}{l}\overleftarrow{ } \\
\infty \\
0 \\
0 \\
0 \\
0\end{array}$ & 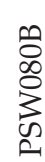 & $\begin{array}{l}\mathbb{3} \\
0 \\
\sum_{0}^{\infty}\end{array}$ & 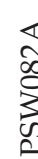 & & 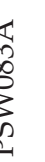 & 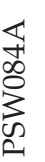 & 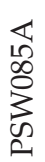 & 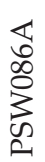 & 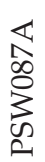 & 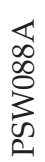 & 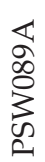 & 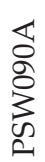 & 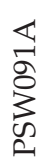 & 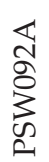 & 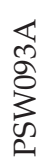 & 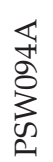 & 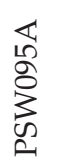 & 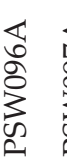 & 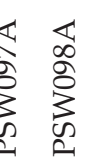 \\
\hline
\end{tabular}




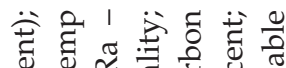
幽

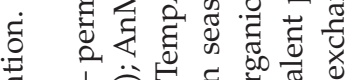
1 穴 5

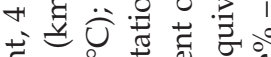

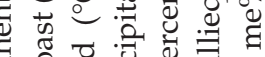
일 过

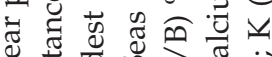

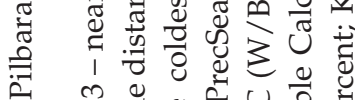

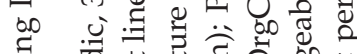
寻 함ㅎㅁ ह वे क्षे

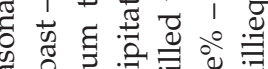
通 $\checkmark$ ) 의 可产

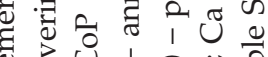

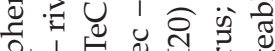
के 1 造 过

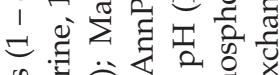
क

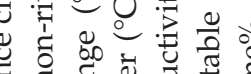
讨

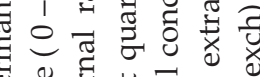

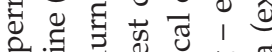
.

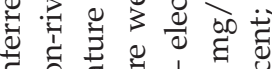

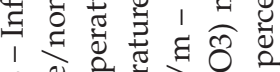
कै है जे वे चु

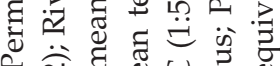
त त्व क्षे 总密

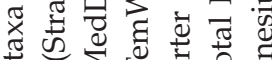
प $\sum$ 造 届

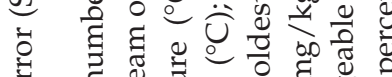

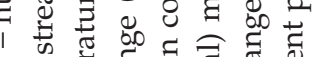

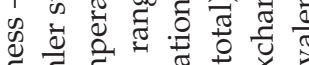

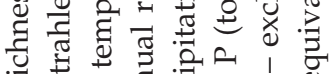

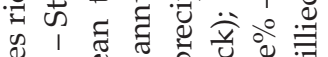
ธैँ कि 항 즌

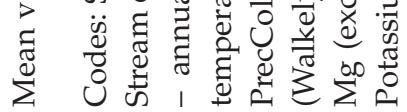

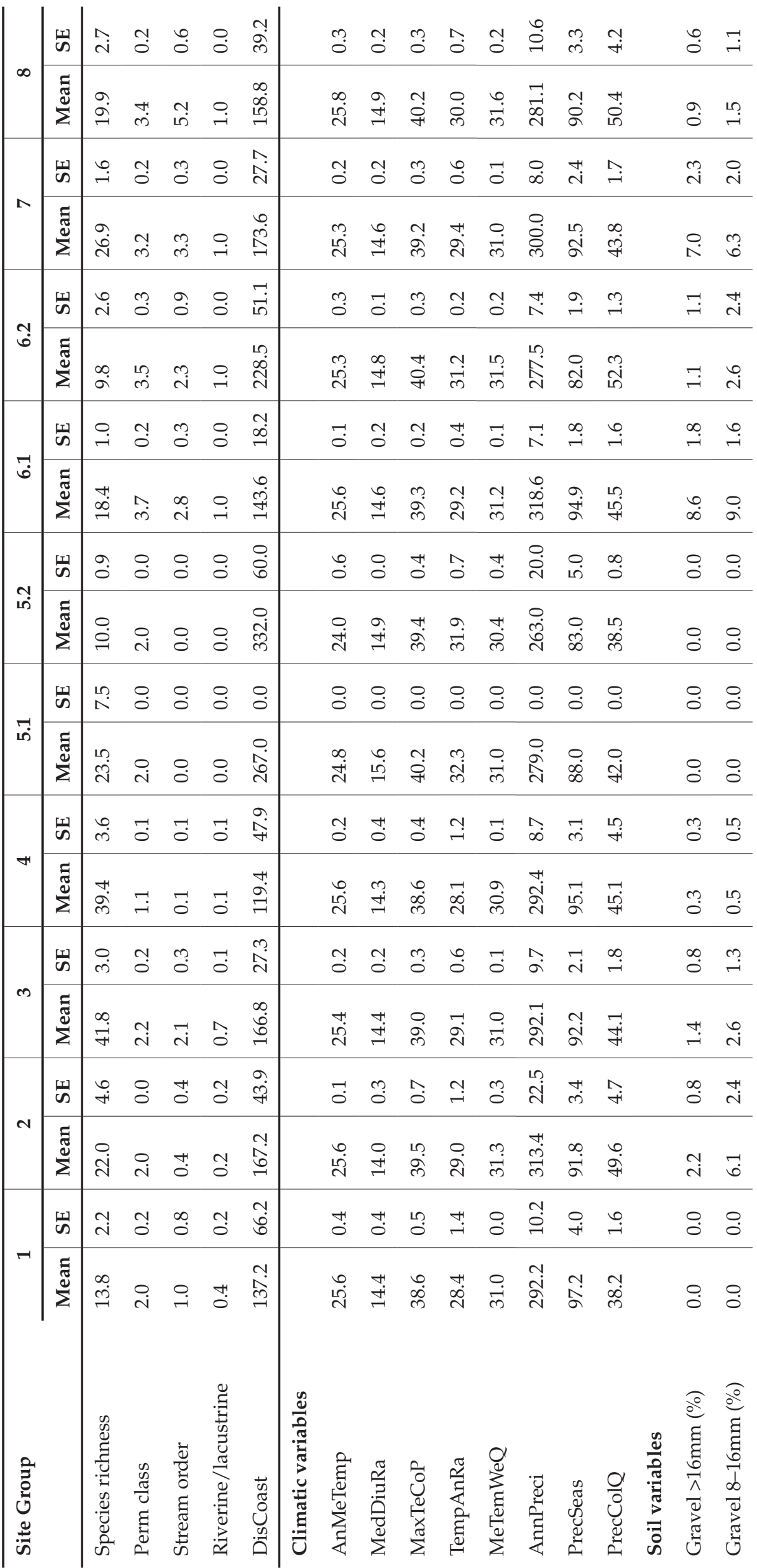




\begin{tabular}{|c|c|c|c|c|c|c|c|c|c|c|c|c|c|c|c|c|}
\hline \multirow[b]{2}{*}{$\infty$} & 离 & $\stackrel{\leftrightarrow !}{\rightarrow}$ & $\stackrel{m}{i}$ & $\overbrace{b}^{3}$ & N & $\stackrel{\text { I }}{+}$ & 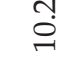 & $\stackrel{3}{0}$ & 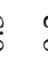 & $\stackrel{1}{0}$ & ন & $\stackrel{m}{i}$ & $\stackrel{\circ}{\circ}$ & $\stackrel{0}{0}$ & ชี & $\overrightarrow{0}$ \\
\hline & $\stackrel{\Xi}{\Sigma}^{\llbracket}$ & $\stackrel{\infty}{-}$ & 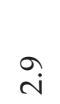 & $\vec{\Omega}$ & $\vec{a}$ & $\stackrel{\infty}{=}$ & $\stackrel{m}{\stackrel{m}{\infty}}$ & $\stackrel{\infty}{\wedge}$ & & $\hat{o}$ & तี่ & $\hat{\circ}$ & மे & $\stackrel{\infty}{i}$ & $\stackrel{0}{0}$ & ț \\
\hline \multirow[b]{2}{*}{1} & 扸 & $\stackrel{+}{\rightarrow}$ & $\stackrel{3}{i}$ & $\stackrel{\circ}{i}$ & $\hat{o}$ & $\stackrel{+}{-}$ & $\stackrel{\overbrace{}}{ \pm}$ & ऽี & 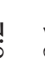 & $\overrightarrow{0}$ & 文 & $\stackrel{\sigma}{\rightarrow}$ & $\stackrel{H}{0}$ & $\stackrel{\text { Lִ }}{0}$ & $\stackrel{0}{0}$ & $\ddot{0}$ \\
\hline & 苂 & $\stackrel{L}{+}$ & $\ddot{a}$ & $\stackrel{\widehat{0}}{\infty}$ & เి & $\stackrel{\curvearrowright}{\wedge}$ & ல் & $\stackrel{0}{0}_{\infty}$ & & $\hat{o}$ & 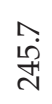 & $\ddot{H}$ & $\stackrel{\infty}{+}$ & $\vec{\phi}$ & $\widetilde{N}$ & $\ddot{0}$ \\
\hline \multirow{2}{*}{ సु. } & 虍 & $\stackrel{m}{i}$ & กู & $\stackrel{\circ}{\sigma}$ & $\overrightarrow{6}$ & $\stackrel{\llcorner}{\infty}$ & تี & ऽุ & & ب. & ले & $\stackrel{H}{\mathrm{~N}}$ & $\stackrel{F}{ت}$ & $\stackrel{\circ}{i}$ & $\stackrel{+}{-}$ & $\stackrel{\text { Lִ }}{0}$ \\
\hline & $\sum^{\text {స్ }}$ & $\stackrel{\llcorner}{\mathrm{i}}$ & $\ddot{b}_{0}$ & $\stackrel{m}{n}$ & $\stackrel{m}{\stackrel{3}{c}}$ & นִ & ڤి & $\widehat{\infty}$ & & $\stackrel{\sim}{\longrightarrow}$ & $\begin{array}{l}0 \\
\stackrel{1}{0} \\
\stackrel{0}{\circ}\end{array}$ & $\stackrel{\llcorner}{\stackrel{\rho}{\wedge}}$ & तै & $\stackrel{+}{\Lambda}$ & $\stackrel{\infty}{\infty}$ & $\stackrel{m}{\sim}$ \\
\hline \multirow[b]{2}{*}{7} & 山ै & กี & $\stackrel{m}{i}$ & $\stackrel{\overbrace{}}{-}$ & $\stackrel{\text { N }}{-}$ & $\hat{o}$ & $\underset{\ddot{\nu}}{\sigma}$ & 5 & & $\tilde{o}$ & $\begin{array}{l}\text { مी } \\
\stackrel{2}{\circ}\end{array}$ & $\stackrel{\infty}{0}$ & $\stackrel{\circ}{\circ}$ & $\stackrel{\infty}{0}$ & $\stackrel{L}{0}$ & ชี \\
\hline & 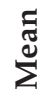 & $\stackrel{\infty}{\stackrel{\oplus}{\ominus}}$ & $\stackrel{m}{\vec{r}}$ & $\begin{array}{l}0 \\
\text { i }\end{array}$ & $\vec{a}$ & $\stackrel{\infty}{\infty}$ & 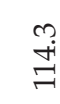 & ${ }_{\infty}^{L}$ & & $\stackrel{\text { Nִ }}{\rightarrow}$ & 峁 & $\begin{array}{l}\infty \\
\stackrel{\infty}{\circ}\end{array}$ & ชู & ت̈ & $\stackrel{L}{\mathrm{~N}}$ & $\stackrel{\infty}{0}$ \\
\hline \multirow{2}{*}{ กี } & 离 & $\stackrel{0}{0}$ & $\stackrel{0}{0}$ & $\stackrel{3}{0}$ & $\stackrel{\circ}{\wedge}$ & $\stackrel{m}{\oplus}$ & मू & - & & $\stackrel{0}{0}$ & ลิ & $\stackrel{+}{i}$ & $\stackrel{?}{\rightarrow}$ & $\stackrel{\longrightarrow}{ت}$ & $\stackrel{\text { Ln }}{O}$ & o. \\
\hline & 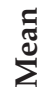 & $\stackrel{0}{0}$ & $\stackrel{\circ}{\circ}$ & 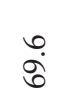 & $\stackrel{\curvearrowright}{\stackrel{a}{-}}$ & $\stackrel{L}{\mathbb{U}}$ & 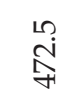 & $\vec{\infty}$ & & $\stackrel{H}{0}$ & $\begin{array}{l}\stackrel{1}{0} \\
\stackrel{\sim}{N}\end{array}$ & $\stackrel{\stackrel{\llcorner}{ }}{\exists}$ & $\stackrel{\text { Ln }}{\stackrel{0}{0}}$ & $\stackrel{\circ}{\dot{m}}$ & $\stackrel{\infty}{\rightarrow}$ & $\stackrel{\text { ì }}{\text {. }}$ \\
\hline \multirow[b]{2}{*}{ in } & 乩 & $\stackrel{0}{0}$ & $\stackrel{\circ}{\circ}$ & $\stackrel{?}{\Lambda}$ & F & $\vec{m}$ & $\stackrel{L}{\mathrm{i}}$ & 5 & & $\stackrel{0}{\circ}$ & ¿ें & $\stackrel{L}{\mathrm{i}}$ & Ḧ. & $\stackrel{m}{0}$ & $\ddot{0}$ & 3 \\
\hline & $\stackrel{\Xi}{\Sigma}^{\Xi}$ & $\stackrel{0}{0}$ & $\stackrel{0}{0}$ & 㟶 & $\stackrel{\infty}{\tilde{\nu}}$ & $\begin{array}{l}\infty \\
\stackrel{\wedge}{0}\end{array}$ & $\begin{array}{l}\text { ப் } \\
\text { I্ }\end{array}$ & $\stackrel{\curvearrowright}{\wedge}$ & & t! & $\begin{array}{l}\stackrel{0}{0} \\
\stackrel{F}{F}\end{array}$ & $\stackrel{\circ}{\circ}$ & ?ై & $\stackrel{\sim}{i}$ & $\stackrel{\infty}{i}$ & 하 \\
\hline \multirow{2}{*}{+} & w & $\stackrel{\circ}{-}$ & $\widehat{\mathrm{i}}$ & $\overrightarrow{\text { ம் }}$ & $\stackrel{\sigma}{-}$ & $\stackrel{\circ}{+}$ & $\hat{\mathrm{i}}$ & : & & $\stackrel{-}{0}$ & 於 & $\hat{\mathrm{N}}$ & $\stackrel{\infty}{\circ}$ & $\stackrel{10}{0}$ & గ̃ & - \\
\hline & 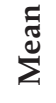 & $\stackrel{\circ}{-}$ & $\stackrel{\infty}{i}$ & $\underset{\infty}{\stackrel{\infty}{\infty}}$ & ํํ & 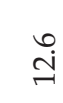 & $\stackrel{m}{=}$ & $\stackrel{10}{\wedge}$ & & ?3. & $\stackrel{\Im}{\mathbb{I}}$ & $\stackrel{\bullet}{\wedge}$ & $\stackrel{M}{i}$ & $\stackrel{+!}{-}$ & $\stackrel{\infty}{\circ}$ & में \\
\hline \multirow{2}{*}{$m$} & 领 & $\stackrel{\circ}{\rightarrow}$ & $\stackrel{\sigma}{ }$ & $\stackrel{\vec{H}}{\vec{H}}$ & $\stackrel{\text { In }}{\rightarrow}$ & $\widehat{\mathrm{i}}$ & $\stackrel{?}{N}$ & - & 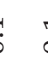 & $\breve{0}$ & 崩 & $\stackrel{\curvearrowright}{-}$ & $\stackrel{\circ}{-}$ & $\ddot{0}$ & $\ddot{0}$ & - \\
\hline & 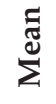 & $\stackrel{\bullet}{-}$ & $\stackrel{m}{m}$ & $\stackrel{0}{\stackrel{\leftarrow}{\Lambda}}$ & $\stackrel{\infty}{\stackrel{\infty}{0}}$ & $\stackrel{\widehat{ }}{\mathrm{A}}$ & సे & $\stackrel{1}{\wedge}$ & & $\hat{o}$ & $\stackrel{0}{\stackrel{0}{\vec{N}}}$ & : & นึ. & $\hat{i}$ & $\stackrel{0}{0}$ & $\stackrel{\infty}{0}$ \\
\hline \multirow{2}{*}{$N$} & w & $\overrightarrow{\ddot{n}}$ & $\stackrel{\bullet}{+}$ & $\begin{array}{l}\infty \\
\infty \\
\infty\end{array}$ & $\widehat{\mathrm{i}}$ & $\overrightarrow{6}$ & $\stackrel{\circ}{\circ}$ & I & 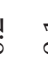 & $\overrightarrow{0}$ & هై & $\stackrel{?}{N}$ & $\stackrel{\infty}{-}$ & $\hat{o}$ & $\overrightarrow{0}$ & $\stackrel{3}{0}$ \\
\hline & 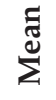 & $\vec{\infty}$ & $\stackrel{\curvearrowright}{ت}$ & $\vec{b}$ & $\begin{array}{l}\infty \\
\stackrel{\infty}{n} \\
\stackrel{\ln }{2}\end{array}$ & $\vec{\infty}$ & $\underset{\sim}{\mathbb{U}}$ & $\overbrace{0}^{\infty}$ & 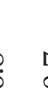 & $\hat{o}$ & $\begin{array}{l}0 \\
\dot{0} \\
\infty\end{array}$ & $\begin{array}{l}\infty \\
\stackrel{\infty}{N}\end{array}$ & $\stackrel{+}{\Lambda}$ & $\stackrel{m}{m}$ & $\stackrel{0}{\circ}$ & $\stackrel{+}{-}$ \\
\hline \multirow{2}{*}{$r$} & 空 & $\stackrel{0}{0}$ & $\stackrel{\circ}{\circ}$ & $\stackrel{0}{\circ}$ & $\stackrel{+}{-}$ & Fi & ت. & ชี & & $\tilde{o}$ & $\overrightarrow{\vec{m}}$ & $\stackrel{19}{\wedge}$ & $\stackrel{\circ}{0}$ & $\hat{o}$ & $\stackrel{m}{0}$ & J \\
\hline & 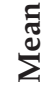 & $\stackrel{0}{\circ}$ & $\stackrel{\circ}{\circ}$ & స్ర & స్ & बें & $\begin{array}{l}\stackrel{\infty}{ } \\
\stackrel{n}{n}\end{array}$ & ف & 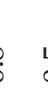 & ᄂ? & $\begin{array}{l}0 \\
\infty \\
\infty \\
\infty \\
\infty\end{array}$ & $\stackrel{\infty}{\stackrel{\infty}{\ominus}}$ & $\stackrel{\infty}{\infty}$ & $\widehat{\mathrm{i}}$ & $\hat{o}$ & $\stackrel{F}{ت}$ \\
\hline 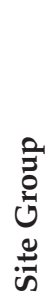 & & 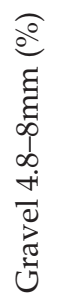 & 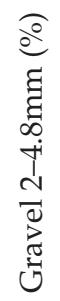 & 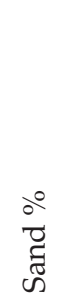 & $\begin{array}{l}\circ \\
\stackrel{\circ}{*} \\
\dot{5 n}\end{array}$ & $\begin{array}{l}\circ \\
\vec{J} \\
0\end{array}$ & 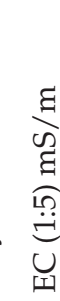 & 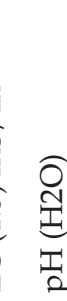 & $\frac{1}{2}$ & 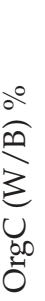 & 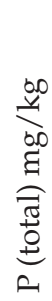 & 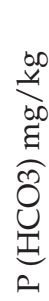 & 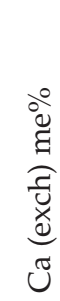 & 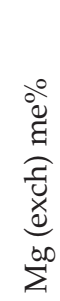 & 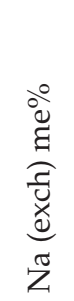 & 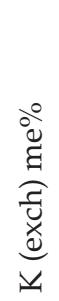 \\
\hline
\end{tabular}




\section{Data analysis}

Species presence/absence data were compiled for sites by combining seasonal samples. Site resemblance matrices were generated using the Bray-Curtis similarity measure in Primer (Primer-E Ltd 2008). The influence of singleton species (species with a single site occurrence) was assessed by comparing resemblance matrices, including and excluding singletons, using Primer's RELATE routine (999 permutations). Analysis of similarity (anosim) routines (Clarke and Green, 1998) were used to examine the significance of relationships between the floristic composition of sites with a priori wetland types, IBRA subregion, permanence, riverine versus lacustrine waterbodies, and catchment basin occurrence. For interpretation, categorical variables were overlain on non-metric multidimensional scaling ordinations of sites (nMDS, 50 starts; Primer-E Ltd. 2008). Two-dimensional ordinations were used as they showed similar stress to 3D outputs.

The BEST/BIOENV procedure in PRIMER was used to explore the relationship between site compositional similarity and environmental attributes. This module uses Spearman's rankorder correlation to match distances in a site association matrix to Euclidean distances among each of its environmental attributes. Continuous variables used in the analysis included chemical and textural attributes of the soil, BIOCLIM climatic variables, distance to coast and altitude. Categorical variables included ordinal permanence class, Strahler stream order and the two-state categorical variable riverine/non riverine. For pairs of variables that were highly intercorrelated $(P>0.9)$, a single variable was retained. In the final data set, distance to coast was retained in preference to annual range of temperature, and percentage clay was retained in preference to percentage sand. Highly skewed variables were log-transformed and the resulting variable matrix normalised.

Some sites were excluded from the analysis of environmental correlates. Soil data were not available for PSW004B, and PSW003A was an extreme compositional outlier. Six saline sites sampling the Fortescue Marsh and Weelarana Salt Lake were also excluded from the BEST/BIOENV procedure since they were compositionally and environmentally very distinct from the remainder of the data set.

\section{Site and species classification}

The data matrix of species presence/absence at sites (excluding singletons) was re-ordered to produce a two-way table for interpretation (Appendix 1) (Belbin 1995) by using the program
PATN (v3.12) to classify sites according to similarities in their species composition (BrayCurtis measure of dissimilarity), and species according to their co-occurrence at sites (the twostep algorithm, Belbin 1980). Both classifications used the flexible UPGMA (unweighted pair-group mean average) sorting strategy with the beta value set to -0.1 (Belbin 1995; Sneath and Sokal 1973). Indicator species showing high fidelity and/ or specificity to site groups were identified using the 'Indicspecies package' in the $\mathrm{R}$ statistical computing language (De Caceres and Legendre 2009, R development core team 2009). Monte Carlo randomisation was used to test the significance of taxa indicative of both individual and multiple groups of sites $(P<0.05)$.

\section{RESULTS}

\section{Sampling adequacy}

Sites included a range of species richness scores (12-52 taxa) and wetland types. Comparison of the observed richness for each site and estimators of total richness (Figure 2 - four examples shown) revealed that the quadrat design employed had captured, on average, $89 \%(79 \%-100 \%)$ of the total richness in the sites (ICE estimator, see Colwell, 2009).

\section{Riparian plant diversity}

A total of 455 taxa was identified from the 98 wetlands and rivers sampled during the survey (Table 1, Appendix 2). These taxa were distributed among 62 families and 202 genera. Dominant plant families were Poaceae $(18.5 \%$ of taxa), Fabaceae $(11.4 \%)$, Asteraceae (10.8\%), and Cyperaceae (8.1\%). Dominant genera included Acacia (4.4\%), Eragrostis (2.9\%), Cyperus (2.9\%), Ptilotus (2.0\%), Eriachne (2.0\%) and Fimbristylis (2.0\%).

The taxa represent approximately $25 \%$ of the known flora of the Pilbara bioregion (S. Dillon unpublished data), and are dominated by species that occur elsewhere in the Eremaean (398 taxa) and Northern (295 taxa) Botanical Provinces (after Beard 1990). Pilbara populations of twenty taxa (e.g. Atalaya hemiglauca, Lobelia arnhemiaca and Terminalia canescens) occur in the Pilbara as populations disjunct from the Kimberley. A group of 143 taxa extend into the South-West Botanical Province. Of these, the majority occur at the periphery of this province, i.e. within the Avon-Wheatbelt (105 taxa) and Geraldton Sandplains (also 105 taxa) bioregions.

Of those taxa with more restricted distributions (known from six or fewer IBRA regions - 88 taxa), only the cosmopolitan Pteris vittata occurs in the 


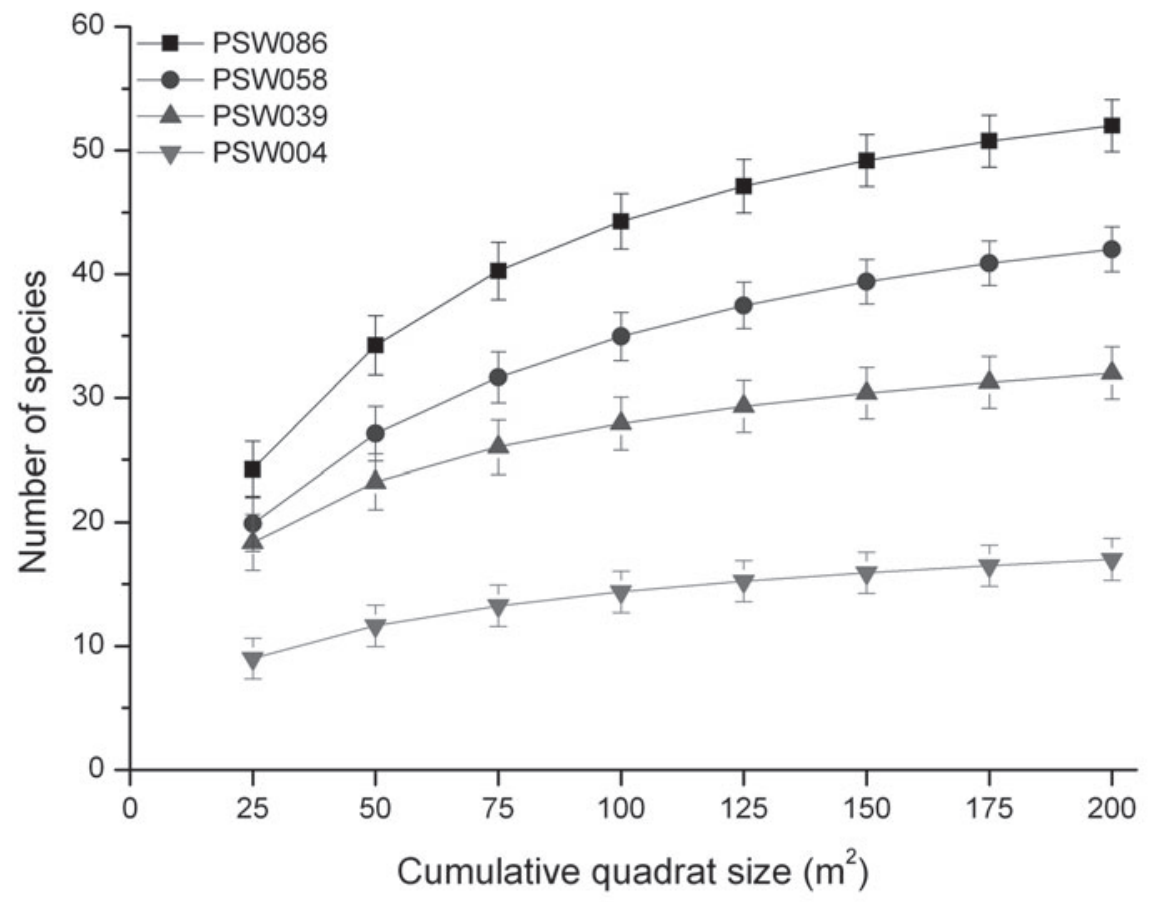

Figure 2 Expected species richness accumulation curves based on the Mao Tau function for four sites with a range of total observed richness. Error bars equal one standard deviation.

temperate south-west of Western Australia. Taxa known from four or fewer bioregions (64 taxa) largely extend into adjacent bioregions. Of these, the Carnarvon (29 taxa) and Gascoyne (29 taxa) share a greater number of taxa with the Pilbara than Dampierland (10 taxa) and the Murchison (nine taxa), with a small component occurring in the adjacent Great Sandy Desert (seven taxa) and Little Sandy Desert (nine taxa) bioregions.

Naturalised taxa represented $6.6 \%$ of the flora, noting that the weed status of some species (e.g. Flaveria trimera) remains contentious (see Keighery 2010). The most frequently recorded weeds included Cenchrus ciliaris ( $46 \%$ of sites), Cynodon dactylon $(22 \%)$, and Cenchrus setiger (17\%). Notable weed records included Conyza parva collected from Chinaman Spring (PSW060), on the northern edge of the study area, a major range extension from near-coastal areas of south-west Western Australia. Previously known anecdotally (Keighery 2010), Gnaphalium polycaulon is confirmed as occurring in the Pilbara, with a record from Coondiner Pool (PSW001).

Sixteen taxa on the Department of Parks and Wildlife's Priority Flora List (Smith 2013) were recorded (see Appendix 2). A number are associated with wetlands within and bordering the Fortescue Marsh Land System as defined by Van Vreeswyk et al. (2004). Both Helichrysum oligochaetum and Rhodanthe ascendens were recorded from
Koondepindawarrina Pool (PSW005). Tecticornia medusa and T. globulifera, formerly known only from the Fortescue Marsh, were also recorded from Weelaranna Salt Lake (PSW043). Eleocharis papillosa, previously known from scattered localities across central Australia and near Onslow at the edge of the Pilbara, was recorded from two sites on the Fortescue Marsh (PSW002, PSW003). The new record of Myriocephalus scalpellus from near Onslow (PSW073) represents the only known population other than the type locality at Coondiner Pool (PSW001). Eremophila spongiocarpa, endemic in the Pilbara and restricted to the Fortescue Marsh, was recorded within its known range (PSW003). Other notable priority taxa include Cladium procerum which occurs in the Pilbara as a major outlier from populations in eastern Australia. Known from both Millstream and Karijini National Park, it was recorded at a known locality (at PSW006). Also recorded from a known population at Fortescue Falls, Adiantum capillus-veneris has a scattering of localised populations across much of Australia. Eragrostis surreyana is endemic in the Pilbara IBRA region, and was recorded from a known population on the Burrup Peninsula. Although not listed as priority taxa, the Pilbara endemics Bergia perennis subsp. perennis, Eriachne tenuiculmis, Sida arsiniata and Stemodia kingii were also recorded during the survey. 


\section{Major range extensions}

The grasses Imperata cylindrica and Pseudoraphis spinescens have distributions that extend from the Kimberley region across northern Australia and down the eastern Australian coast to South Australia, with numerous inland occurrences. Not previously known from the Pilbara, Imperata cylindrica occurred at three sites: Fortescue Falls (PSW006), Palm Spring on Cave Creek (PSW009) and Millstream Delta (PSW011). Prior to the current survey, the only Pilbara record of Pseudoraphis spinescens was a collection by A. Forrest on the Fortescue River in 1878 (Mueller 1881a, 1881b). During the survey it was collected at four sites: Moreton Pool (PSW030), Munreemya Billabong (PSW036), Curara Claypan (PSW072), and West Peawah Creek Pool (PSW079). Nymphoides indica, recorded at De Grey claypan (PSW035), is the first collection for the Pilbara bioregion. Its known distribution includes much of the world's tropics and extends from the Kimberley across the north and down the east coast of Australia.

\section{Species frequency and richness}

Of the 450 taxa analysed, two-thirds (66.4\%) occurred at fewer than five sites (see Appendix 1 ), and one-third (33.2\%) occurred at a single site (Appendix 3). Three taxa, Cyperus vaginatus (perennial sedge), Eragrostis tenellula (annual grass) and Centipeda minima (annual herb), occurred at more than half the sites. Species richness ranged from three (PSW032) to 87 taxa per site (PSW085) and averaged $26.3 \pm 1.4$. Richness showed significant differences between wetland types (Kruskal-Wallace 1-way ANOVA, $P<0.005)$, with gorge creeks $(20.0 \pm 2)$, springs $(18.3 \pm 1.4)$ and salt marshes $(14.5 \pm 3.5)$, showing the lowest richness. Gorge creeks were characterised by massive rock and provided limited habitats for annual taxa, instead being dominated by dense perennial sedges and shrubs in soil pockets that are perennially moist. Springs were commonly dominated by dense patches of perennial sedges (typically Cyperus vaginatus), often interspersed with bare areas of calcrete. Salt marsh sites had a limited suite of halophytic taxa.

Rock pools $(42.5 \pm 2.5)$, clay flats $(41.0 \pm 6)$, clay pans $(29.7 \pm 3.2)$ and ephemeral creeks (28.7 \pm 4.4 ) had the highest mean richness with rich assemblages of herbs and annual and perennial grasses.

Richness also showed significant differences between inferred permanence classes (KruskalWallace 1-way ANOVA, $P<0.005)$. Ephemeral sites had the highest mean species richness $(39.2 \pm 3.6)$ and permanent sites the lowest $(20.8 \pm 2.4)$. This is related largely to the relative proportion of different wetland types within each class. Ephemeral sites included a number of rock pools (PSW071A, PSW080A) with herb-rich aprons, and claypans and clay flats with rich assemblages of annual taxa. Permanent sites included river pools (15 sites), gorge creeks and the majority of species-poor springs (13 of 15). Seasonal and near-permanent sites showed little difference in richness and were dominated by river pools. Little difference in richness was evident for riverine sites in relation to Strahler stream order; with only order-one versus order-three showing significant pairwise differences $(P<0.05)$.

Richness of rare taxa, including singletons and species occurring at fewer than six sites, was examined for geographic patterns (Figure 3). Concentration of rare taxa was evident within the Fortescue Marsh and on the periphery of the study area at a small number of sites which included Weelarrana Salt Marsh (PSW043B), claypans, granite rocks and a single seasonal creek (PSW085) at the southern edge of the region. Some concentration of rare taxa along the northern edge of the Hamersley IBRA subregion is associated with rarely recorded taxa from the gorges and upland ephemeral creeks of the Hamersley Range. Notably, few singleton and rare taxa were observed in the Chichester IBRA subregion (Figure 3). High numbers of rare taxa were associated with both wetland-type and water permanence. Overall, ephemeral and seasonal wetland types such as salt marshes, rock pools and claypans showed highest numbers of rare taxa, and their non-uniform distribution across the study area explains much of the observed geographical patterning.

\section{Site classification}

Comparison of the full and reduced (singletons excluded) species presence/absence matrices revealed that the two were highly correlated (Rho $=0.99, \mathrm{p}<0.001)$. In preference, the reduced matrix was used.

The primary division in the classification largely separated non-turbid riverine sites from predominantly non-riverine wetlands and turbid riverine sites with fine-textured soils. Within the last major group, the small number of saline sites formed a distinct group at the next division in the classification. The site classification was ultimately examined at the 8-group level (Figure 4). The tengroup classification and a priori wetland types overlain on 2D nMDS ordinations (Figure $5 \mathrm{~A}$ and $\mathrm{B})$, revealed a strong correspondence between floristic groupings and wetland type. 

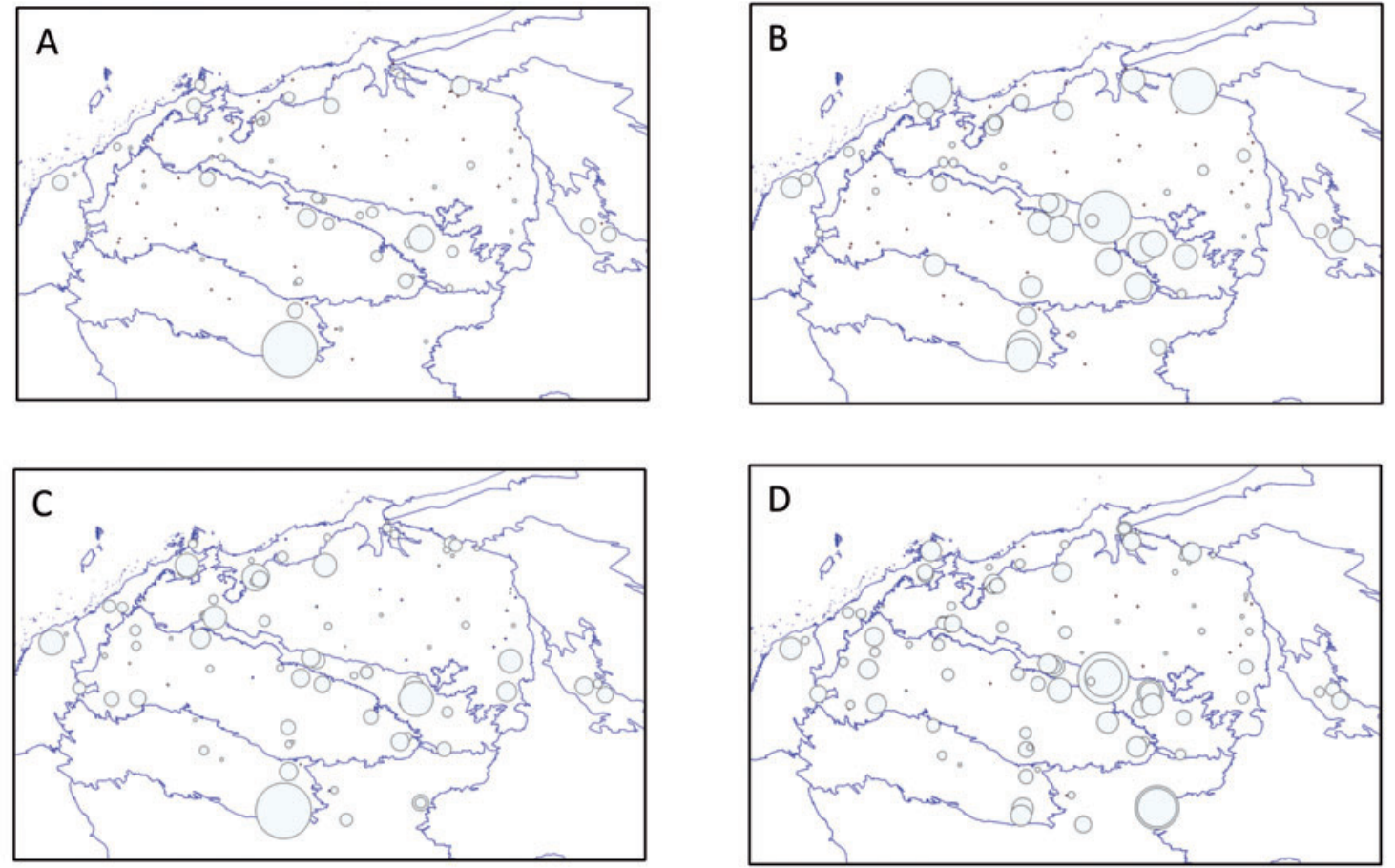

Figure 3 Number of rare taxa (single site record) or uncommon taxa $(\leq 5$ site records) recorded per site, with symbol-size proportional to richness. (A) Richness within sites containing rare taxa (maximum 15 taxa). (B) Richness within sites containing rare taxa as a proportion of total site richness (maximum 0.27). (C) Richness within sites containing uncommon taxa (maximum 31). (D) Richness within sites containing uncommon taxa as a proportion of total site richness (0.87). Sites with no rare taxa are solid dots.

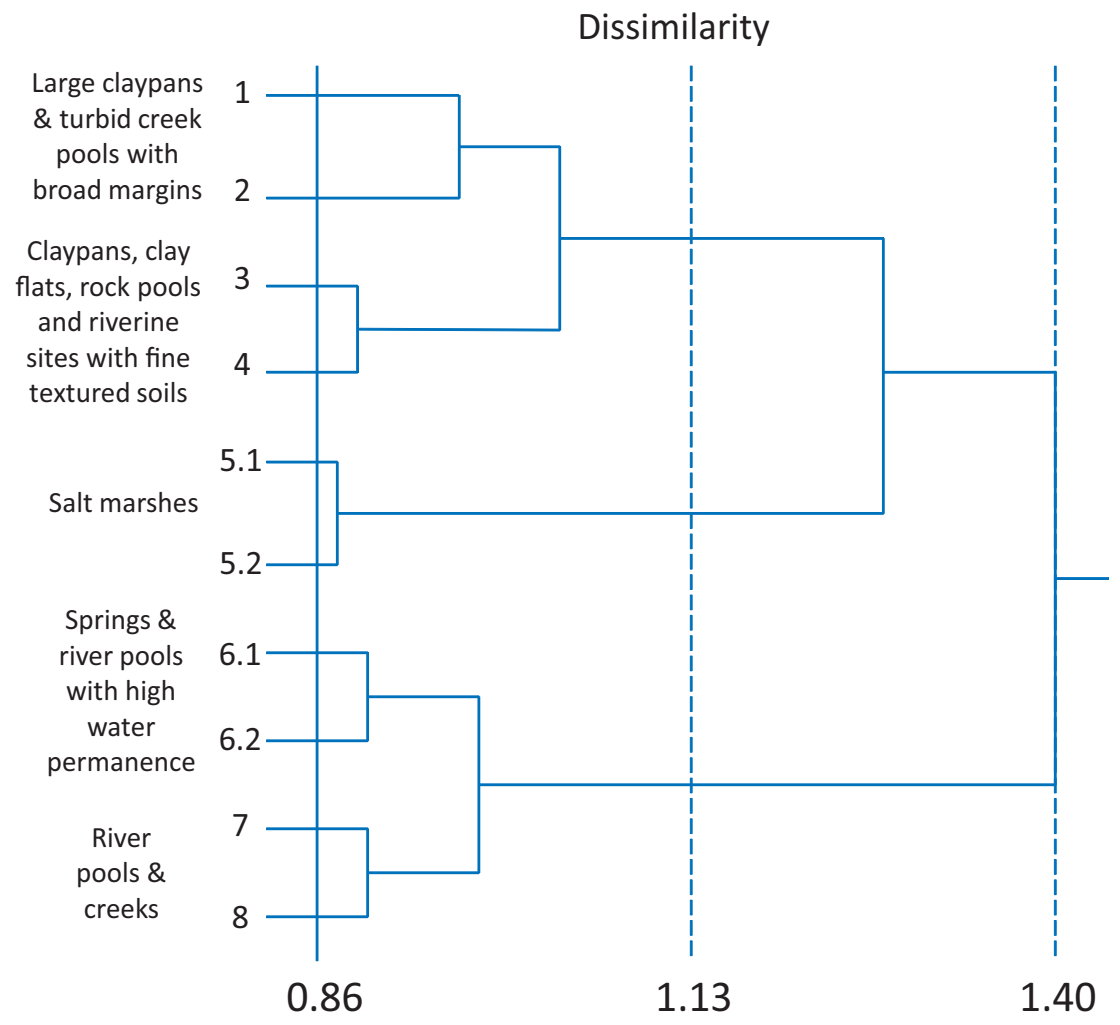

Figure 4 Classification of 103 survey sites (UPGMA) based on species presence/absence. Classification is shown at the eight group level with descriptions summarising divisions in the classification. 


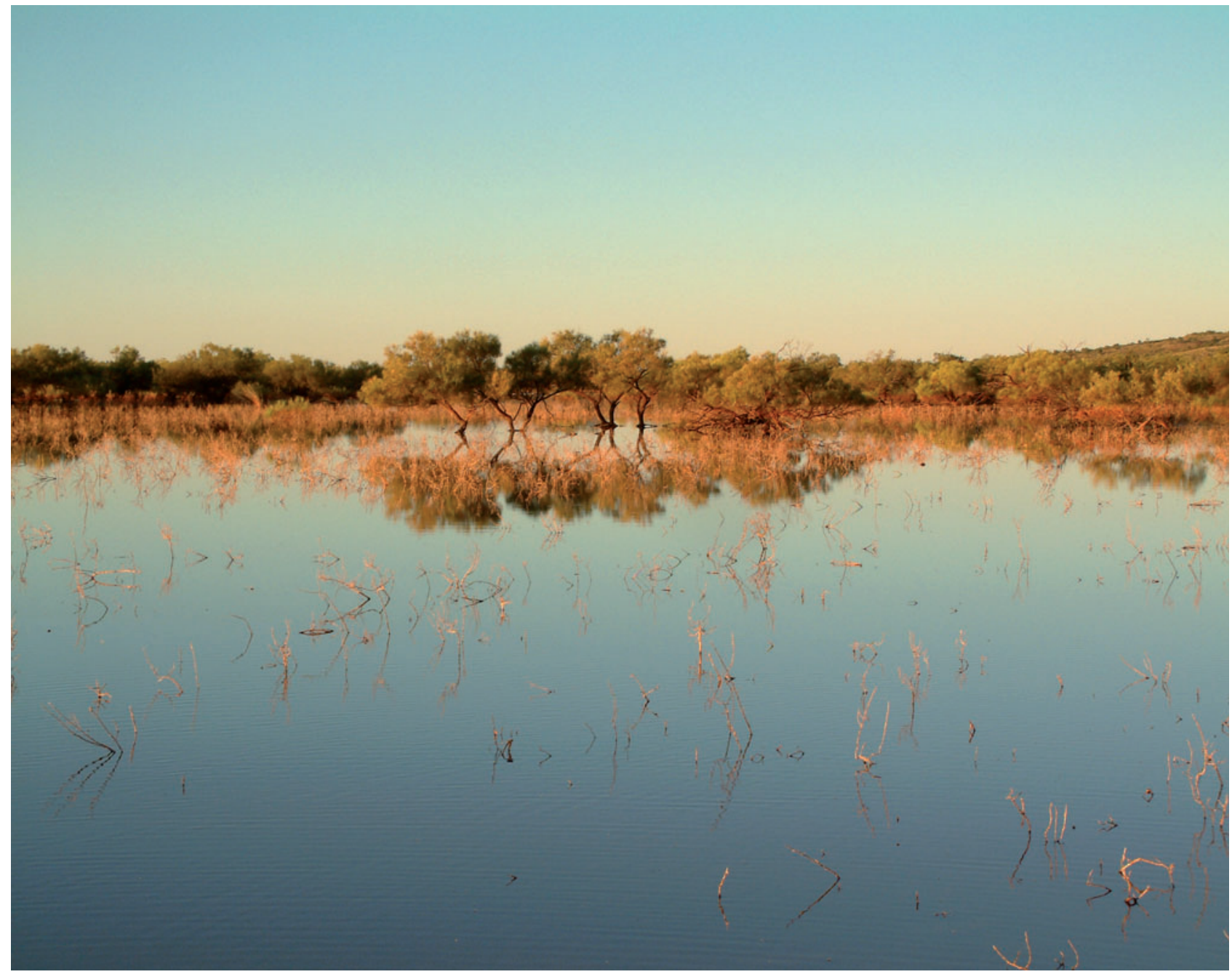

Plate 4 Large seasonal/episodic claypan on Mulga Downs Station (site PSW040A). Stands of Acacia stenophylla occur across the inundated bed and within the riparian zone.

\section{Large claypans and turbid linear pools with broad margins}

Group 1. A group of five claypan sites at four wetlands including large claypans and a single linear turbid claypan (Coondiner Pool), all typified by very broad, low-gradient margins. Structural dominants within the group included scattered Eucalyptus victrix over Eriachne benthamii with Marsilea spp. forming dense swards. Significant indicator species included Schoenoplectus laevis, Elytrophorus spicatus, Eriocaulon cinereum, Nymphoides indica, Glossostigma diandrum and Rotala diandra. Sites sampled showed a high percentage of clay content $(26.9 \pm 4.1)$ and the lowest EC $(5.8 \pm 1.1)$ and $\mathrm{pH}(6.8 \pm 1.1)$ of all site groups. These sites, with fine-textured soils, also had high total phosphorus $(388.0 \pm 131.9 \mathrm{mg} / \mathrm{kg})$. The few wetlands in the group were scattered from the north-west coastal margin to the inland eastern edge of the study area and had the lowest species richness of groups 1-4 (13.8 taxa per site).
Group 2. A group of five sites at four wetlands including three claypans and the margin of a linear, turbid pool. This group also featured Eucalyptus victrix as a structural dominant over a relatively species-rich herb layer. Significant indicator species included Sporobolus mitchellii, Bergia trimera, B. perennis, Myriocephalus oldfieldii, M. rudallii and Muehlenbeckia florulenta. The sites showed similar soil chemistry attributes to group 1 (Table 2).

Claypans, clay flats, rock pools, and riverine sites with fine-textured soils

Group 3. A large group of 23 sites with the highest species richness of all site groups (41.8 taxa per site). Sites sampled the riparian zones of linear turbid creeks, river pools, ephemeral creeks, claypans and clay flats (Table 1). Although including a number of wetland morphologies, sites within the group shared fine-textured soils in both riverine and non-riverine settings (mean silt $10.8 \pm 1.5 \%$ and clay $17.7 \pm 2.7 \%$ ). Seventeen sites 
included Eucalyptus victrix or E. camaldulensis subsp. refulgens as overstorey trees within the riparian zone. Analysis revealed a single taxon, Fimbristylis littoralis, as the significant indicator species for the group. The group shared a number of taxa with group 4 (see below) with Fimbristylis microcarya, Wahlenbergia tumidifructa, Lipocarpha microcephala, Oldenlandia galioides and Drosera indica being significant indicator species for both site groups taken together.

Group 4. A group of seven sites on the margins of claypans and the aprons of rock pools. As a group, the sites were close to the coast relative to all other groups $(119.4 \pm 47.7 \mathrm{~km})$. Claypans in the group were small, highly ephemeral, and occurred within Triodia-dominated flats and dune systems. The edges of pans were low sandy rises with a narrow band of annual taxa recruiting after rainfall and wetland-filling. The group showed similarly high species richness to group 3 (39.4 taxa per site). Sixteen herbs and annual grasses were significant indicator species for the group with Synaptantha tillaeacea var. tillaeacea, Eriachne aristidea, Bulbostylis barbata, Calandrinia ptychosperma, Cyperus pulchellus and Gomphrena leptoclada subsp. leptoclada significant at $\mathrm{P}<0.05$.

\section{Salt marshes}

Group 5.1. A subgroup of two sites within the upper fringe of the Fortescue Marsh, characterised by a group of salt-tolerant indicator species including Cressa australis, Muellerolimon salicorniaceum, Cyperus bulbosus and Eleocharis papillosa. The sites showed high EC $(145 \pm 2.5)$ and phosphorus (total $\mathrm{P}, 410 \pm 20$ ) and high clay content $(27.8 \pm 3.1)$. The current survey, however, did not attempt to capture the full floristic variation evident within and bordering the Fortescue Marsh. These two sites sample an extensive zone within the Fortescue Marsh that is dominated structurally by Muellerolimon salicorniaceum.

Group 5.2. A subgroup comprising four sites at two wetlands, sampling the edges of the main basins of the Fortescue Marsh and Weelarrana Salt Lake. They were characterised by low shrublands dominated by Tecticornia spp. Significant indicator species for the subgroup included Tecticornia halocnemoides sens. lat., T. indica, T. medusa, T. globulifera and Triglochin hexagona. The sites showed the highest soil EC $(472.5 \pm 98.4)$ of all groups, and high total P $(267.5 \pm 27.7 \mathrm{mg} / \mathrm{kg})$. Species richness was low with 10 taxa per site. The sites are likely to experience significant periods of inundation during major flooding events.
Springs and river pools with high water permanence

Group 6.1. A subgroup of 23 sites from river pools and 11 of the 15 springs sampled. Sites had a mean species richness of 18.4 taxa, the significant indicator species being Schoenoplectus subulatus and Fimbristylis sieberiana. Structural dominants included Melaleuca argentea, Eucalyptus camaldulensis subsp. refulgens, Acacia ampliceps, Typha domingensis and Cyperus vaginatus. The group also included all the sampled springs and gorges within Karijini and Millstream National Parks. The group had the highest mean permanence score (3.7) of all site groups. This was coupled with the highest mean annual rainfall $(318.6 \mathrm{~mm} / \mathrm{yr})$. The soils of the sites contained the highest percentage gravel (all fractions) and were otherwise typically sandy. Exchangeable calcium was the highest of all groups (6.2 me\%) and is likely to reflect the contribution of calcium-rich groundwater to the riparian zone substrate.

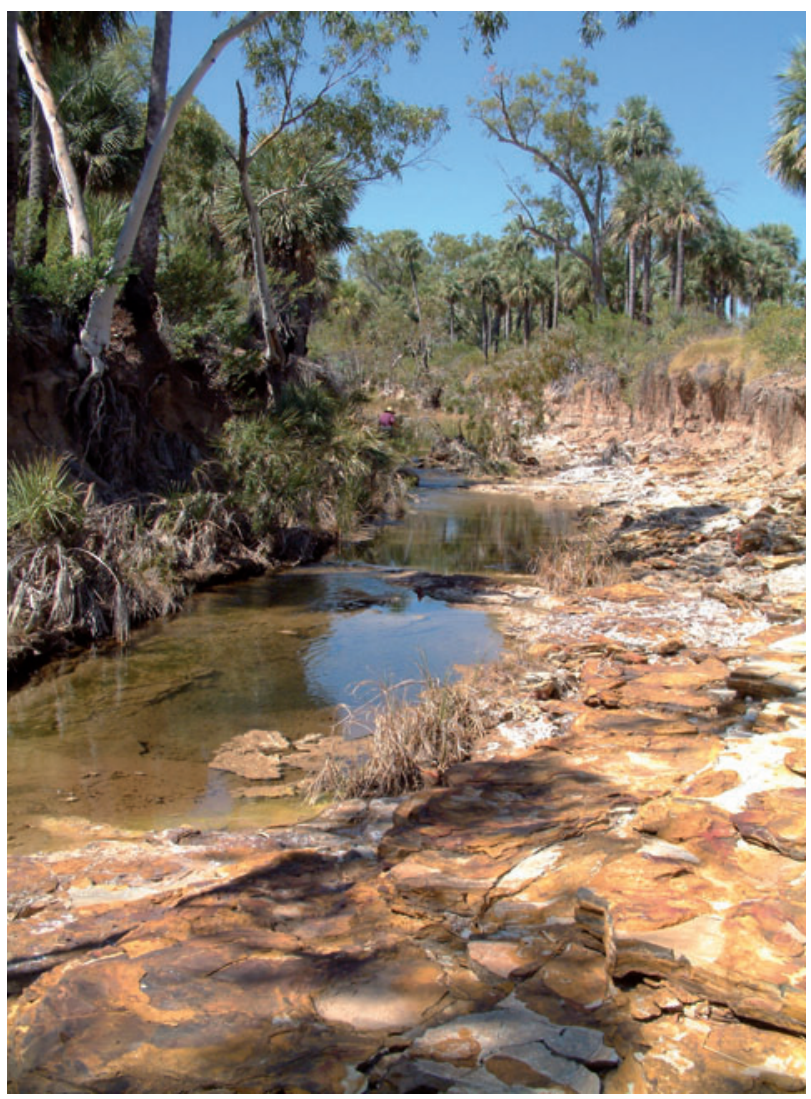

Plate 5 Areas of bedrock and calcrete dominate the margins of Palm Spring (site PSW013A). Melaleuca glomerata, juvenile Livistona alfredii (Millstream fan-palm) and sedges occur in the shallow soil pockets along the spring-fed creek (M.N. Lyons). 
Group 6.2. A subgroup of four species-poor sites (9.8 taxa per site) comprising two river pools and two small springs. Structural dominants included Acacia ampliceps and Cyperus vaginatus. Sites showed significant cattle disturbance or flood damage, which probably accounted for their low species richness scores. Stylobasium spathulatum and the naturalised taxon Bidens bipinnata were significant indicator species for the group.

\section{River pools and creeks}

Group 7. A group of 21 riverine sites including 15 clear river pools, four ephemeral creeks and two springs. The group shows compositional similarities to subgroup 6.1 yet showed higher mean species richness (26.9 cf. 18.4 taxa per site). The group had a mean permanence score of 3.1 (cf. 3.7 for group 6.1), reflecting the small number of springs and inclusion of ephemeral creeks within the group. Indicator species analysis did not reveal any taxon to be individually significant for the group. Eucalyptus camaldulensis subsp. refulgens and Cyperus vaginatus are structural dominants. The separation of groups 6.1 and 7 is due largely to the very low frequency of taxa dependent on high/ permanent moisture, such as Melaleuca argentea, Schoenoplectus subulatus and Typha domingensis within group 7, and the presence of a group of widespread annual taxa including Eragrostis tenellula, Wahlenbergia tumidifructa, and Centipeda minima that are largely absent from group 6.1. Soil texture attributes of groups 6.1 and 7 were very similar (Table 2).

Group 8. A group of nine sites on permanent and near-permanent higher-order river pools structurally dominated by Melaleuca argentea, Eucalyptus camaldulensis subsp. refulgens, Cyperus vaginatus and Cynodon dactylon. Average species richness was lower than the other group dominated by river pools (19.9 taxa per site, cf. 26.9 in group 7). Soils within the group were more finely textured than groups 6.1 and 7, with lower gravel fractions (Table 2).

Plate 6 Pool in ephemeral creek (site PSW062A) at the base of the Gregory Range. The riparian zone is dominated by Eucalyptus victrix and Cyperus vaginatus, with Schoenoplectus subulatus in the waterbody (N. Gibson).

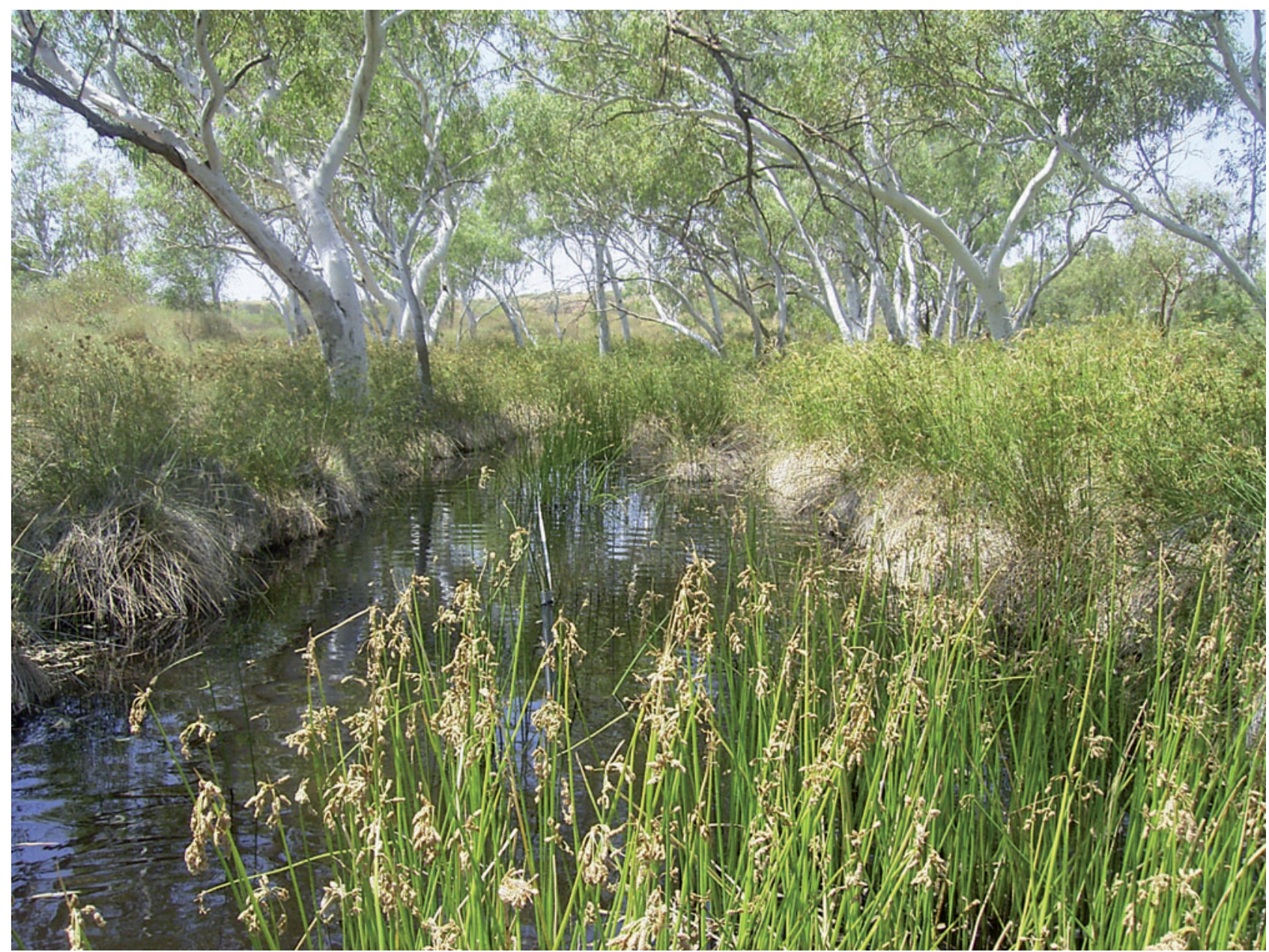



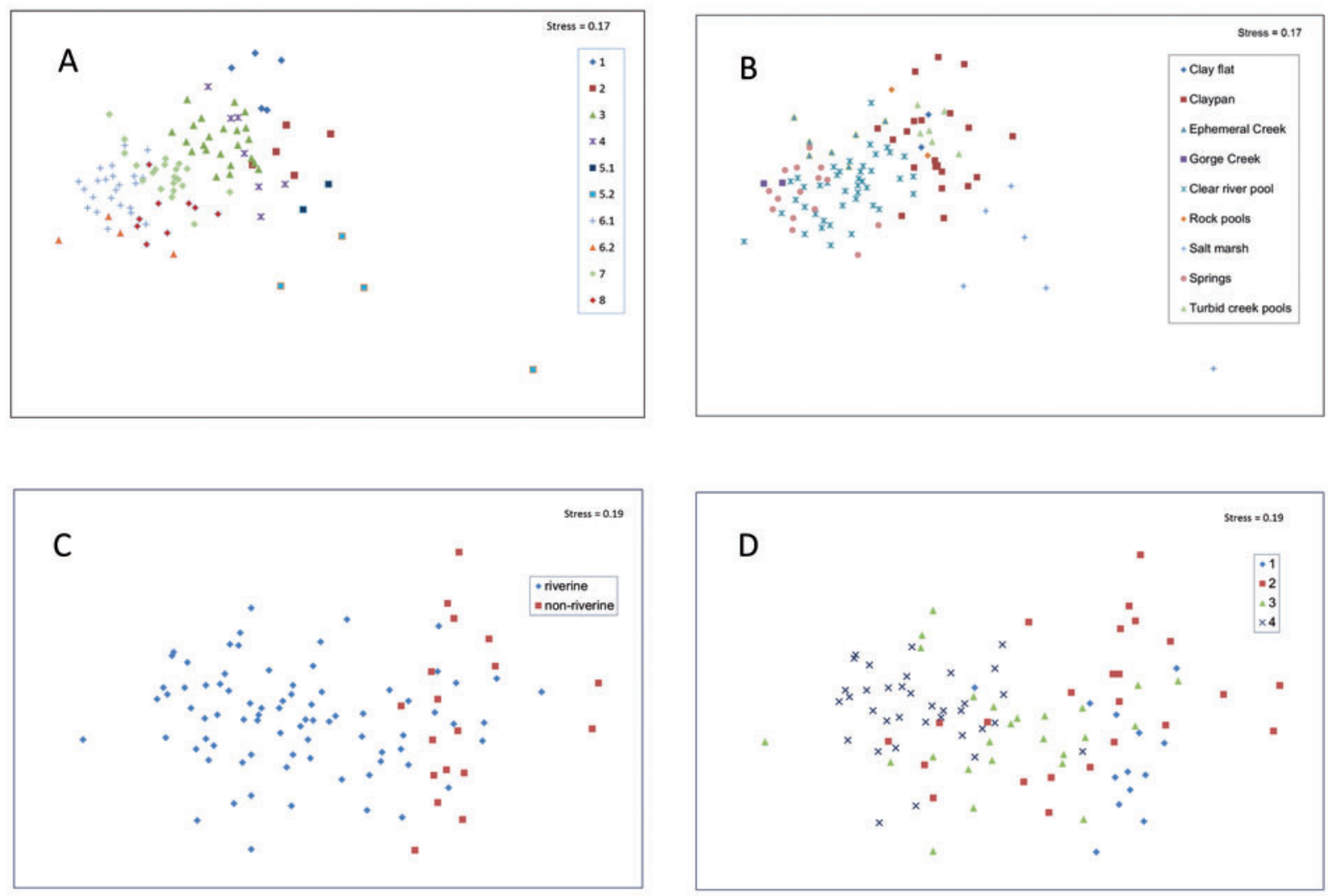

Figure 5 Two dimensional. non-metric multi-dimensional scaling ordination (2D nMDS) of 103 sites based on presence / absence of plant species overlain with (A) floristic groups and (B) a priori wetland types. Also, 2D nMDS ordination excluding salt marshes (floristic subgroups 5.1 and 5.2) with sites coded (C) as riverine/non-riverine and (D) according to permanence class (1, ephemeral; 2, seasonal episodic; 3, near permanent; 4 , permanent).

\section{Environmental variables and floristic composition}

Significant compositional differences were observed for riverine versus non-riverine sites (Figure 5C) $($ Rho $=0.525)$, and permanence class (Figure $5 \mathrm{D})(\mathrm{Rho}=0.383)$. Within riverine sites, excluding the very small number of ephemeral sites, permanent sites were compositionally different from seasonal and near-permanent sites $($ Rho $=0.25)$, although there was no significant difference between stream orders (Rho $=0.022$ ). Geographic patterns were examined by testing compositional differences against IBRA subregions and major drainage basins. IBRA sub-regions showed only moderate compositional differences $($ Rho $=0.241)$, with pairwise differences between both the Fortescue and Roebourne Plain against all other sub-regions accounting for the bulk of the patterning. Little support for compositional differences between drainage basins was observed $($ Rho $=0.073)$.
The BEST procedure revealed that a combination of five variables [riverine/non-riverine, DisCoast, clay\%, EC (1:5), $\mathrm{pH}$ ] provided the highest rank correlation with the site dissimilarity matrix (Spearman's rho $=0.53, \mathrm{P}<0.01$ ). The inclusion of the categorical variable riverine/non-riverine confirms the major compositional separation of these two major wetland classes. The soil variables relate to a number of compositional gradients. High clay content corresponds to the separation of clear riverine sites with relatively coarse sediments, from claypans and turbid riverine sites typified by finely textured soils. The latter were compositionally distinct with relatively rich assemblages of annual taxa. The inclusion of soil electrical conductivity and $\mathrm{pH}$ also correlates with the compositional separation of riverine and non-riverine sites. The inclusion of DisCoast reflects the greater occurrence of a number of compositionally distinct groups along the subdued landscapes of the coastal plains of the study area, including claypans and turbid creek pools. 


\section{DISCUSSION}

\section{Flora and diversity}

Prior to the current study, the only regional survey of the Pilbara riparian flora was undertaken by Masini (1998) and Masini and Walker (1989) as two separate studies sampling subsections of the region. Although interrupted by cyclonic activity and subsequent sampling late in the dry season, the authors recorded $c a .300$ taxa. Many localised surveys assessing impacts of proposed mining developments have also been completed (e.g. Biota Environmental Sciences, 2012). Other studies have been undertaken to assess the impacts on Pilbara wetlands of water abstraction and mine dewatering and its disposal (e.g. Johnson and Wright, 2001; Braimbridge et al. 2010). The only other regionalscale wetland floristic survey undertaken in Western Australia's arid zone was completed by Gibson et al. (2000), in the southern half of the Carnarvon Basin bioregion to the south of the Pilbara. They recorded 263 taxa from 57 wetlands (albeit with smaller quadrat size) representing ca.12\% of the region's flora (Keighery et al. 2000).

Gibson et al. (2000) observed that the wetland flora of the southern Carnarvon Basin, while dominated by taxa of the arid zone (Eremaean), had affinities with both temperate and tropical floras. In part, this is true of the Pilbara, although the temperate component of the flora is smaller and largely composed of very widespread taxa rather than those with temperate distributions for which the Pilbara is their northern range limit. The Pilbara riparian flora also includes a larger component of the tropical flora, from both the rocky landscapes of the northern Kimberley and the more topographically subdued central Kimberley, Ord-Victoria Plain and Victoria-Bonaparte bioregions. A subset of these does not occur elsewhere in the Eremaean areas of Western Australia (e.g. Atalaya hemiglauca). Notable elements of the tropical riparian flora are also near their southern limit in the Pilbara (e.g. Melaleuca argentea and Sesbania formosa).

While sharing broadly similar climates (Leighton, 2004; Wyrwoll et al. 2000), the strong affinities of the Pilbara flora with the adjoining Carnarvon and Gascoyne bioregions are also attributable to the regions possessing a similar suite of wetland types. All share a combination of riverine and non-riverine water bodies that represent similar hydrological settings. Indeed some of the major structural dominants in riverine riparian zones are common to the three regions (e.g. Eucalyptus camaldulensis subsp. refulgens and Acacia coriacea subsp. pendens). In contrast, the sandy deserts to the east and northeast of the Pilbara are characterised by internal drainage with few major riverine habitats. Where these occur they are commonly broad, sometimes saline, drainage lines flowing into basins, or highly ephemeral creek systems arising in isolated ranges. The saline coastal areas of the Pilbara and the Fortescue Marsh share taxa with these desert systems (e.g. Tecticornia auriculata). The deserts, like the Pilbara, contain a suite of claypans and clay flats that are episodically inundated and elements of their flora are shared.

The proportion of uncommon taxa recorded for the Pilbara - singletons (33.2\%) and those from fewer than five sites $(66.4 \%)$ - is of similar magnitude to other similar studies. Gibson et al. (2000) recorded that $55 \%$ of taxa occurred at a single site (singletons) in the southern Carnarvon Basin. A more comprehensive survey of temperate zone wetlands in Western Australia showed very similar proportions ( $31 \%$ and $59.3 \%$ respectively) to the current study (Lyons et al. 2004). The occurrence of this uncommon component of the flora (at similar levels) is also a consistent feature of quadrat-based floristic surveys carried out across the wider Western Australian landscape (Gibson et al. 2004; Keighery et al. 2000; Lyons et al. 2014), and poses problems for designing reserve systems that aim to capture major floristic communities.

Species richness of sites was related to both the a priori wetland types and the site groups derived from the classification based on floristic composition. Maximum richness occurred in less permanent wetlands, with concomitant higher richness in non-riverine sites. A similar pattern was observed for the proportion of uncommon taxa across the range of wetland types. Coarsely textured soils that limit moisture retention, as well as major disturbance associated with the high energy/ volume flows following flood events, are likely to limit the suite of taxa that can persist along many riverine riparian zones in the Pilbara. The plant communities of these larger riverine sites are often simple, with groundwater-dependent overstorey trees (Melaleuca argentea, Eucalyptus camaldulensis subsp. refulgens) (O'Grady 2006) and simple understoreys of shrubs, rhizomatous grasses and sedges that are more resistant to flood waters (e.g. Melaleuca linophylla, Cyperus vaginatus). In contrast, more ephemeral sites, those with lower flow-energy (e.g. turbid linear creeks), as well as claypans, clay flats and rock pools, experience much-reduced flood disturbance and their soils are more finely textured. In many of these shallow wetlands, the wet/dry ecotone is broad, providing habitats for a larger range of taxa with differing responses to wetting and drying (Brock and Casanova 1997). The development of species-rich seed banks that persist 
for long periods between fill events has also been shown to contribute to the richness of these sites (Brock et al. 2006).

The extensive aquifer systems of the Pilbara (Waters and Rivers Commission 1996) discharge groundwater to form permanently flowing creeks, typically in rocky sites, or contribute flows to permanent pools within river channels. Springs form a group that is relatively poor in species (18.3 taxa per site) in the Pilbara, and are characterised by taxa requiring permanently damp or wet soil that physically dominated sites, e.g. Cyperus vaginatus, Melaleuca argentea, Typha domingensis. Studies of springs elsewhere in Australia confirm that springs of the Pilbara are floristically distinct from the remainder of the continent. In the Kimberley and tropical Northern Territory, rainforest floristic elements are a major component of spring floras (Kenneally et al. 1991; Russell-Smith 1991). Detailed studies of the springs of the Great Artesian Basin (Fensham and Fairfax 2003; Fensham and Price 2004), while very different floristically from the Pilbara, highlight the importance of springs in the conservation of endemic and relictual taxa. In the Pilbara, springs and spring-fed river pools and associated gorge habitats provide important mesic refugia for taxa such as Livistona alfredii, Stylidium weeliwolli and Fimbristylis sieberiana, including major distributional outliers such as Cladium procerum, Phragmites karka, Imperata cylindrica and Adiantum capillus-veneris. In arid Central Australia, permanent water bodies, including springs and seepages, have also been shown to be important refugia for relict and endemic species (White et al. 2000; Brim Box et al. 2008).

\section{Floristic composition}

Patterns in the floristic composition of Pilbara wetlands and rivers correspond to major hydrological and substrate attributes of the sites. These environmental differences are largely embodied by the initial a priori site groups that influenced the sampling design and are reflected in the site groups I derived.

The vegetation of the Fortescue Marsh is compositionally distinct from the remainder of the site groups, even though sampling of the Marsh was limited relative to its complexity and size. While I did not sample tidal environments along the Pilbara coast that are likely to share some taxa with the Marsh (e.g. Tecticornia indica) it contains a floristically unique element of the Pilbara wetland flora.

Among the remaining rivers and wetlands a major compositional separation is seen between riverine and non-riverine (wetland) sites. Turbid creek pools, while riverine, show clear floristic similarities to claypans. Turbid sites are a feature of creeks and smaller rivers in Pilbara lowlands. Like claypans, they have fine soils, have generally short hydroperiods and are not subject to major flood flows. This is reflected in the analysis with soil texture emerging as a significant term in the BEST analysis. Masini (1988) also highlighted the importance of sediment texture and water permanence in structuring Pilbara wetland plant communities, finding strong correspondence between morphological/hydrological (subjective) and floristic (objective) classification of sites. Importantly, both the current study and that of Masini (1988) are very site-specific. Dryland rivers show considerable heterogeneity in terms of channel morphology and sediment attributes along their length, particularly where over-bank flows fill secondary channels (Thoms et al. 2006). As a consequence, even a small stretch of river $(<5 \mathrm{~km})$ may capture a number of floristic elements.

Biogeographic patterns in the riparian flora across the Pilbara are explained by gross differences in the distribution of major wetland types across its four IBRA subregions. This is not surprising, given that, relative to the broader landscape, riparian zones are somewhat buffered from the dominant climatic gradient that occurs from west to east in the region, particularly in terms of moisture availability (Leighton, 2004). In particular, at the regional scale, spring sites are largely decoupled from the prevailing climate. The topographically subdued, largely depositional landscapes of the Fortescue and Roebourne Plains IBRA sub-regions show significant differences from the remaining sub-regions.

\section{Conclusions}

The Pilbara riparian flora represents $25 \%$ of the Pilbara flora, and is dominated by Eremaean and tropical taxa, with strongest affinities to adjoining externally draining bioregions.

Biogeographic patterning is limited, with sub-regional differences being related to the relative proportion of wetland types within each subregion. Riverine sites, in particular, show limited compositional difference across the region. The Fortescue valley, coastal plains and other lowland areas make important contributions to the diversity of non-riverine wetlands. Claypans and clay flats capture a large component of the Pilbara wetland flora, and their scattered occurrence across the lowlands of the region poses difficulties in capturing their diversity in the reserve system. Within the region, the Fortescue Marsh, along with spring sites in Karijini and Millstream National Parks, support unique elements of the Pilbara riparian flora. 


\section{ACKNOWLEDGEMENTS}

I thank David Mickle for his unwavering assistance and companionship in the field and office. Field work was assisted by Neil Gibson, Natalia Huang and Simon Lyons. Adrian Pinder led the site selection with valuable initial input from Peter Kendrick and Stephen van Leeuwen. Pilbara DEC staff provided welcome accommodation and delivered much-needed parts for broken field equipment. Numerous pastoralists and indigenous communities provided local knowledge, hospitality and access to their lands. Adrian Pinder drafted Figure 1. Steve Dillon provided his Pilbara species list and Ben Richardson provided data on the species IBRA occurrences. Lesley Gibson assisted with indicator species analysis. Norm McKenzie along with two anonymous referees provided valuable comments on the draft manuscript.

\section{REFERENCES}

Anderson, M.J., Gorley, R.N. and Clarke, K.R. (2008). Plymouth: Primer-E; 2008. PERMANOVA+ for PRIMER: Guide to software and statistical methods.

Beard, J.S. (1990). Plant life of Western Australia. Kangaroo Press: Kenthurst, Australia.

Belbin, L. (1980). TWOSTEP: A program incorporating asymmetric comparisons that uses two steps to produce a dissimilarity matrix. Technical Memorandum 80/9, CSIRO Division of Land Use Research: Canberra, Australia.

Belbin, L. (1995). PATN technical reference. CSIRO Division of Wildlife and Ecology: Canberra, Australia.

Biota Environmental Sciences (2012). A vegetation and flora survey of the Koodaideri study area. Unpublished report prepared for Rio Tinto: Perth, Australia. (online: http://www.epa.wa.gov.au/EIA/referralofPropschemes / Lists / Proposal / Attachments / 178 / Appendix\%204_A\%20Vegetation $\% 20$ and $\% 20$ Flora\%20Survey.pdf)

Braimbridge, M., Antao, M. and Loomes, R. (2010). Groundwater dependent ecosystems for Millstream: ecological values and issues, Environmental water report series, Report no. 13, Department of Water, Government of Western Australia.

Brim Box, J., Duguid, A., Read, R.E., Kimber, R.G., Knapton, A., Davis, J. and Bowland, A.E. (2008). Central Australian waterbodies: The importance of permanence in a desert landscape. Journal of Arid Environments 72: 1395-1413.

Brock, M., Capon S.J. and Porter, J.L. (2006). Disturbance of plant communities of desert rivers (pp 100-132). In: Kingsford, R. (ed.), Ecology of desert rivers. Cambridge University Press: Cambridge, UK.

Brock, M. and Casanova, M.T. (1997). Plant life at the edge of wetlands: ecological responses to wetting and drying (pp. 181-192). In: Klomp, N. and Lunt, I. (eds), Frontiers in ecology: Building the links. Elsevier Science: Oxford, UK.

Clarke, K.R. and Green, R.H. (1988). Statistical design and analysis for a 'biological effects' study. Marine Ecology Progress Series 92: 205-219.
Colwell, R.K. (2009). EstimateS: Statistical estimation of species richness and shared species from samples. Version 8.2. User's Guide and application, published at http://purl.oclc.org/estimates

De Caceres, M. and Legendre, P. (2009). Associations between species and groups of sites: indices and statistical inference. Ecology 90: 3566-3574.

Fensham, R.J. and Fairfax, R.J. (2003). Spring wetlands of the Great Artesian Basin, Queensland, Australia. Wetlands Ecology and Management 11: 343-362.

Fensham, R.J., Fairfax, R.J. and Sharpe, P.R. (2004). Spring wetlands in seasonally arid Queensland, environmental relations, classification and conservation values. Australian Journal of Botany 52: 583-595.

Geological Survey of Western Australia (1990). Geology and mineral resources of Western Australia. Memoir 3. Western Australian Geological Survey: Perth, Australia.

Gibson, N.G., Keighery, G.J. and Lyons, M.N. (2000). The flora and vegetation of the seasonal and perennial wetlands of the southern Carnarvon Basin, Western Australia. Records of the Western Australian Museum, Supplement 61: 175-216.

Gibson, N., Keighery, G.J., Lyons, M.N. and Webb, A. (2004). Terrestrial flora and vegetation of the Western Australian wheatbelt. Records of the Western Australian Museum, Supplement 67: 139-189.

Houlder, D.J., Hutchinson, M.F., Nix, H.A. and McMahon, J.P. (2000). ANUCLIM User Guide, Version 5.1. Centre for Resource and Environmental Studies, Australian National University: Canberra, Australia.

Johnson, S.L. and Wright, A.H. (2001). Central Pilbara groundwater study. Hydrological Record Series, Report HG 8. Waters and Rivers Commission: Perth, Australia.

Keighery, G.J. (2010). The naturalised vascular plants of the Pilbara region, Western Australia. Records of the Western Australian Museum, Supplement 78: 299-311.

Keighery, G.J., Gibson, N., Lyons M.N. and Burbidge, A.H. (2000). Flora and vegetation of the southern Carnarvon Basin. Records of the Western Australian Museum, Supplement 61: 77-154.

Kenneally, K.F., Keighery, G.J. and Hyland, B.P.M. (1991). Floristics and phytogeography of Kimberley rainforests (pp 91-131). In: McKenzie, N.L., Johnston, R.B. and Kendrick, P.G. (eds), Kimberley rainforests of Australia. Surrey Beatty and Sons: Chipping-Norton, Australia.

Leighton, K.A. (2004). Climate (pp 19-38). In: van Vreeswyk, A.M.E., Payne, A.L., Leighton, K.A. and Hennig, P. (eds) (2004). An inventory and condition survey of the Pilbara region, Western Australia. Technical Bulletin No. 92. Western Australian Department of Agriculture: Perth, Australia.

Lyons, M.N., Gibson, N., Keighery, G.J. and Lyons, S.D. (2004). Wetland flora and vegetation of the Western Australian wheatbelt. Records of the Western Australian Museum, Supplement 67: 39-89.

Lyons, M.N., Keighery, G.J., Gibson, L.A. and Handasyde, T. (2014). Flora and vegetation communities of selected islands off the Kimberley coast of Western Australia. Records of the Western Australian Museum, Supplement 81: 205-243.

McKenzie, N.L., van Leeuwen, S. and Pinder, A.M. (2009). Introduction to the Pilbara Biodiversity Survey, 
2002-2007. Records of the Western Australian Museum, Supplement 78: 3-89.

Masini, R.J. (1988). Inland waters of the Pilbara Western Australia (Part 1), A report of a field study carried out in March-April 1983. Technical Series 10, Environmental Protection Authority Perth, Western Australia.

Masini, R.J. and Walker, B.A. (1989). Inland waters of the Pilbara Western Australia (Part 2), A report of a field study carried out in October-November 1984. Technical Series 24, Environmental Protection Authority: Perth, Western Australia.

Meissner, R., Owen, G. and Bayliss, B. (2009). Flora and vegetation of the banded iron formation of the Yilgarn Craton: Robinson Range and Mount Gould. Conservation Science Western Australia 7: 363-376.

Mueller, F. (1881a). Plants of north-western Australia. Votes and Proceedings of the Legislative Council during the first session of 1881, no. 1. Government Printer: Perth, Australia.

Mueller, F. (1881b). A catalogue of plants collected during Mr. Alexander Forrest's geographical exploration of North-west Australia in 1879. Journal and Proceedings of the Royal Society of New South Wales 14: 81-95.

Pinder, A.M., Halse, S.A., Shiel, R.J. and McRae, J.M. (2010). An arid zone awash with diversity: patterns in the distribution of aquatic invertebrates in the Pilbara region of Western Australia. Records of the Western Australian Museum, Supplement 78: 205-246.

R Development Core Team (2009). R: A language and environment for statistical computing. R Foundation for Statistical Computing: Vienna, Austria.

Russell-Smith, J. (1991). Classification, species richness, and environmental relations of monsoon rainforest in northern Australia. Journal of Vegetation Science 2: 259-278.

Smith, M.G. (2013). Threatened and priority flora list for Western Australia. Department of Parks and Wildlife: Kensington, Western Australia.

Sneath, P.H.A. and Sokal, R.R. (1973). Numerical taxonomy: the principles and practice of numerical classification.
Freeman: San Francisco, USA.

Strahler, A.N. (1952). Hypsometric (area-altitude) analysis of erosional topography. Bulletin of the Geological Society of America 63: 1117-1142.

Thackway, R. and Cresswell, I.D. (1995). An interim biogeographic regionalisation of Australia. Australian Nature Conservation Agency: Canberra, Australia.

Thoms, M.C., Beyer, P.J. and Rogers, K.H. (2006). Variability, complexity, and diversity: the geomorphology of river ecosystems in dryland regions (pp 47-75). In: Kingsford, R.T. (ed), Ecology of desert rivers. Cambridge University Press: Cambridge, UK.

van Vreeswyk, A.M.E., Payne, A.L., Leighton, K.A. and Hennig, P. (eds) (2004). An inventory and condition survey of the Pilbara region, Western Australia. Technical Bulletin No. 92. Western Australian Department of Agriculture: Perth, Australia.

Walshe, T.V., Halse, S.A., McKenzie N.L. and Gibson, N. (2004). Towards identification of an efficient set of natural diversity recovery catchments in the Western Australian Wheatbelt. Records of the Western Australian Museum, Supplement 67: 365-384.

Waters and Rivers Commission (1996). Pilbara region water resources: review and development plan, summary report 4 . Western Australian Waters and Rivers Commission: Perth, Australia.

White, M., Albrecht, A., Duguid, A., Latz, A., Hamilton, M., Latz, P. and White, M. (2000). Plant species and sites of botanical significance in the southern bioregions of the Northern Territory; vol. 1: Significant vascular plants. Report to the Australian Heritage Commission from the Arid Lands Environment Centre, and the Parks and Wildlife Commission of the Northern Territory: Alice Springs, Australia.

Wyrwoll, K.-H., Courtney, J. and Sandercock, P. (2000). The climatic environment of the Carnarvon Basin, Western Australia. Records of the Western Australian Museum, Supplement 61: 13-27.

\section{APPENDIX 1 [ELECTRONIC]}

Site by species data matrix, reordered according to their site and species classifications. The floristic groups are indicated. Taxa recorded from one site are excluded (see Electronic Appendix 3).

\section{APPENDIX 2 [ELECTRONIC]}

List of taxa recorded from 103 sites sampled during the survey (superscript ${ }^{1}$ denotes pairs of infraspecific taxa analysed at specific rank, superscript ${ }^{2}$ denotes closely related taxa that could not be reliably discriminated and were amalgamated for analysis). Numbers following taxa are Department of Parks and Wildlife priority flora codes (see Smith 2013).

\section{APPENDIX 3 [ELECTRONIC]}

Site by singletons species data matrix.

\section{See CD inside the back cover or visit}

http://www.museum.wa.gov.au/research/records-supplements/ 